\title{
A Hierarchical Multi-Resolution Agent Based Modeling and Simulation Framework for Household Electricity Demand Profile
}

\author{
Imran Mahmood ${ }^{1,2}$, Quair-tul-ain', Hasan Arshad Nasir'², Fahad Javed ${ }^{2}$ and José A. Aguado ${ }^{3}$ \\ 1 Department of Computer Science, College of Engineering Design and Physical Sciences, Brunel University, Kingston \\ Lane, Uxbridge, London, UB8 3PH, UK \\ 2 Center for Research in Modeling and Simulation (CRIMSON), School of Electrical Engineering and Computer Science \\ (SEECS), National University of Sciences and Technology (NUST), Islamabad, Pakistan \\ 3 Department of Electrical Engineering, Universidad de Malaga, Malaga Spain \\ * Correspondence: imran.mahmood@ seecs.edu.pk
}

\begin{abstract}
Analyzing demand behavior of end consumers is pivotal in long term energy planning. Various models exist for simulating household load profiles to cater different purposes. A macroscopic viewpoint necessitates modeling of a large-scale population at an aggregate level, whereas a microscopic perspective requires to measure loads at the granular level, pertinent to the individual device of a household. Both aspects have lucrative benefits, thus ignites the need to combine them into a modeling framework to allow model scalability and flexibility, and to analyze the domestic electricity consumption at different resolutions. In this applied research, we propose a multi-resolution Agent based Modeling and Simulation framework for the estimation of domestic electricity consumption. Our proposed framework simulates per minute electricity consumption by combining the large neighborhoods, behavior of household individuals, their interactions with the electrical appliances, their sociological habits and the effects of exogenous conditions such as weather and seasons. In comparison with the existing energy models, our framework uniquely provides a hierarchical, multi-scale, multiresolution implementation using a multi-layer architecture. This allows the modelers a flexibility to model largescale neighborhoods at one end, without the loss of expressiveness in modeling microscopic details of individuals' activities at house level and energy consumption at the appliance level, at the other end. The validity of our framework is demonstrated using a case study of 264 houses. A validated ABMS framework will support: (i)Effective energy planning; (ii) Estimation of the future energy demand; (iii) and the Analysis of the complex dynamic behaviour of the consumers.
\end{abstract}

Keywords: Agent based Modeling and Simulation; Multi-resolution modeling; Electricity Demand Profiles; Electricity consumption behaviour; Urban Electricity Use; Occupancy Patterns; Electricity Appliances; Model Validation

\section{Nomenclature}

$\mathbf{P}_{\text {start }} \quad$ Starting probability of an appliance

A Appliance

$\Delta \mathbf{t}_{\text {comp }} \quad$ Computational Timestamp

h Hour

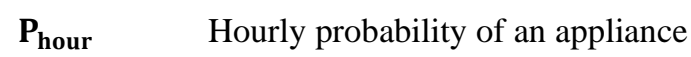

f Mean daily starting frequency of each Appliance

d Day of Week

$\mathbf{P}_{\text {step }} \quad$ Step size of the scaling factor that scales the probabilities according to $\Delta \boldsymbol{t}_{\boldsymbol{c o m p}}$

$\mathbf{P}_{\text {sat }} \quad$ Saturation probability of the appliances 


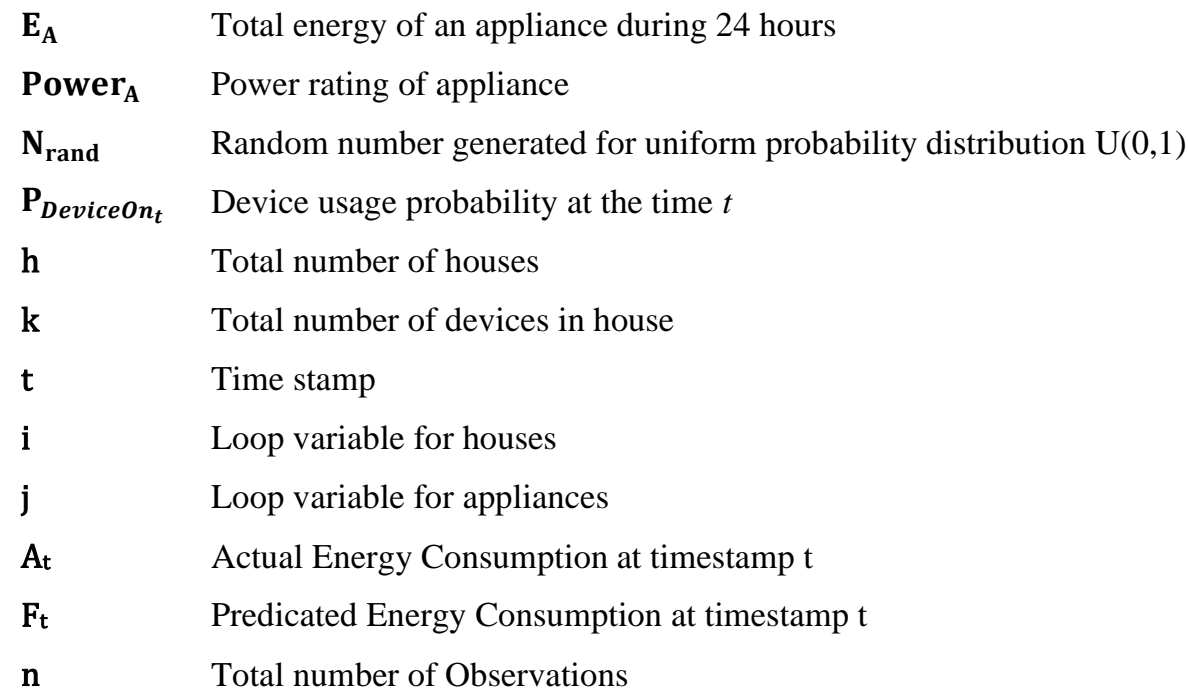

\section{Introduction}

The continuous rise in the rate of population growth and rapid urbanization are the key causes to severe power crisis, especially in a developing country. Frequent blackouts lasting for hours, power failures, inadequate expansion of installed capacity, inefficiencies in power transmission, line losses, among others are the most ominous signs of these energy crisis. As per World Energy Council, the average household electricity consumption in different countries lies between $570-11,879 \mathrm{kWh} /$ year. Due to its prominence, the household consumer sector is gaining an increasing attention of the research community, with impetus for greater efficiency, control and transparency, through demand response management, IoT technologies, smart grid infrastructure and renewable integration. In the developing world, this interest is even more pronounced (1). To meet the current load requirements and cope with the future rising demand of electricity, development of sustainable policies through proper planning is becoming highly desirable, yet increasingly challenging. In order to meet long-term targets for a sustainable, efficient, affordable and low carbon energy system, a descriptive study of the energy consumption dynamics at a country scale is inevitably necessary (2) (3). The descriptive view helps reveal insights and analyze synthetic demand profiles over time. This allows the stakeholders and the decision makes to observe the dynamic behavior of different consumer sectors in the country, and assist them in the effective decision making in the integrated energy planning of a country.

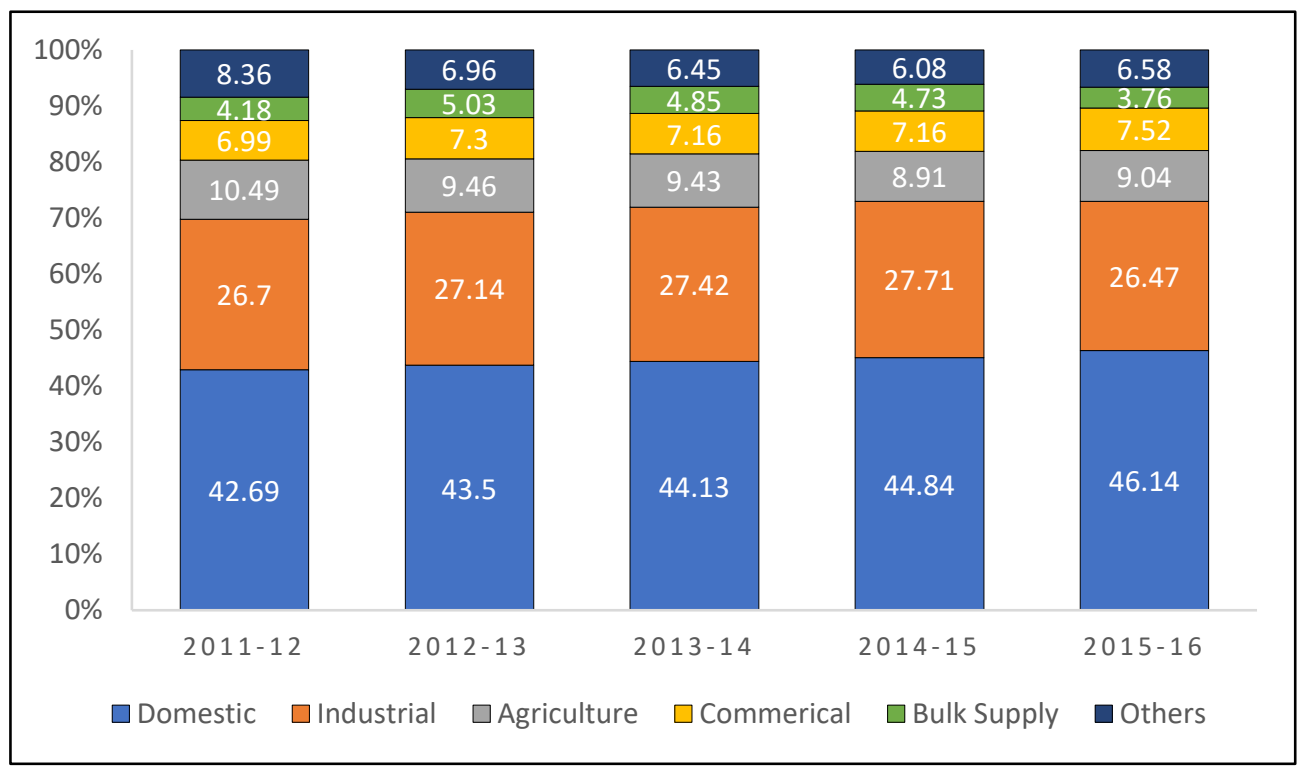

Figure 1: Mean electricity \% consumption in Pakistan 
The predictable and repetitive patterns of hourly electricity demand and historic load curves of major consumer sectors, such as: (i) Domestic (ii) Industrial (iii) Commercial and (iv) Agricultural, are fundamentals to explore. Particularly, the domestic sector, which tends to be extremely volatile, unpredictable, and highly dynamic (3). Household electricity usage is mainly influenced by lifestyles, socio-demographics, economic policies, electric appliances, building characteristics, weather conditions, residential heating and cooling technologies, and many others (4). Therefore, household load forecasting is considered to be quite complex in nature (5). The mean electricity percent sale in Pakistan of different consumer classes is shown in Figure 1. It is evident from the figure that the major consumer class is domestic which constitutes more than $40 \%$ of the total electricity sale, and is increasing yearly (6). We therefore position the focus of this paper on the domestic consumers.

Energy system modelling serves as the basis for making strategic decisions, where a significant driving factor in the energy system dynamics is the electricity demand, commonly obtained using empirical datasets and through the simulation of energy consumption processes. Majority of the models focus on the annual consumption by simply scaling the historic load curve. Consequently, they disregard the dynamic evolution of consumption patterns of short timescales, that are vital for studying the balance of demand and supply (7). A standard load profile is a representative curve that is used to predict the estimated demand for specific consumers, obtained from measurements. Synthetic (or simulated) load profiles, on the other hand, are based on representative samples and the heterogeneous dynamic behavior of the specific consumers, consumer groups or appliances. It is obtained from statistical analysis or bottom up simulation models (8). It is desirable to deploy a dynamic electricity demand model to investigate the behavioral dynamics of the energy system under varying conditions, at short time scales, and with consumers having complex heterogeneous behavior. All of that cannot be easily be described by simply aggregating the individual entities (9).

Modeling and simulation enable us to replicate a real-world system for risk-free dynamic experiments, which are otherwise impossible, dangerous or costly and time consuming. ABM is a paradigm meant for simulating the actions and interactions of autonomous agents, abstracting both individual or collective entities, with a view to assessing their effects on the system as a whole (10). Applications of ABM provide abstraction of real systems, at a microscopic level to model and explore complex behavior over time (11). Microscopic models help in simulating real-world problems with higher resolution, as these consider granular details, that can produce minor deviations from the actual result if not considered (12). An ABM is the composition of individual agents and environment. Each agent individually assesses its situation and makes decision on basis of certain set of rules. Every agent has its own goals and have some knowledge processing abilities. The emergent behavior resulted from the individual behaviors and interactions between these agents affects the aggregated level of the total system. ABM technique is used by many researchers as suitable approach for complex socio-technical problems; particularly to study the wholesale electricity markets (13). However, recently the framework is used for modeling of smart grid scenarios such as demand response strategies, distributed generation, integration of renewable technologies and energy storage (13).

In this paper, we focus on the application of the ABM paradigm to model the energy consumption of a typical region in an urban area of a country and using a hierarchical, multi-scale, multi-resolution agent-based modeling and simulation framework. There are several advantages associated with the properties of our proposed framework:

(i) Hierarchical: Complexity usually takes the form of a hierarchy, where a complex system is composed of interrelated subsystems that have their own subsystems, until the lowest elementary level is reached. The architecture of a complex system is a function of its components and their hierarchic relationships. The main advantage of hierarchical modeling is the reduction of complexity through the logical decomposition (14). We conceptualize the electricity consumption sector as a decomposable hierarchic structure, which is composed of different entity types: Neighborhood, House, Occupants, Appliances. Each of these entity types are linked with each other in a hierarchal relationship. The details of our proposed hierarchy are given in section 4;

(ii) Multiscale: In Modeling \& Simulation, multiscale modeling refers to the simultaneous use of different scales to describe a system of time and/or space. The key advance of this approach is the flexibility our framework allows in running the simulator at varied scales (15). It can be configured to run on minute, hour, day, week, month or year basis and allows the coverage of any spatial region from a single street to as large as a city or province, depending on the computational power of the platform. 
(iii) Multiresolution: In modeling, resolution model refers to the level of details captured in the process of abstraction while conceptualizing the real system. The greater details lead to higher resolution of the model. In ABM, resolution plays an important role as it brings a tradeoff between higher level of details and the ability to populate a large number of agents. A multi-resolution modeling framework accommodates different levels of details of different interacting entities simultaneously within the same framework.

Our proposed framework uses a multi-layered architecture to support multi-resolution modeling at a time, including (a) Macro-level: to model a scalable population of households; (b) Meso-level: to model different groups of occupants and their sociological features; and (c) Micro level: to model individual appliances and their energy consumption behaviour at a granular level. Our proposed framework offers a flexibility of modeling large scale neighborhoods at one end, without the loss of expressiveness in modeling microscopic details of individuals' activities at house level and energy consumption at the appliance level, on the other end. In our proposed framework, the challenges of spatio-temporal resolution, stochastic nature of consumer's behavior, integration of complex human behavior and sociologic factors, interdisciplinary modeling and scalability are covered, through a hierarchical, multi-scale, multiresolution implementation. It also utilizes the stochastic superimposition of different exogenous influencing factors such as weather, seasonality and the device usage pattern. The aggregated load of all the devices for each hour is compared with the actual load. The simulation results of hourly coefficient of variation of the root mean square error CV(RMSE) are within the $30 \%$ threshold as defined in ASHRAE Guidelines (16).

In the absence of smart meters in Pakistan, it is quite difficult to estimate the dynamic daily electricity demand in the domestic sector. Our approach presents a dynamic simulator which estimates the load profile of a domestic neighborhood based on an initial model configuration and different input parameters. We provide a 'proof of concept' using NUST university housing data and demonstrate its usefulness. Such simulator with customizable settings is currently not available to the local industry and hence is taken as a valuable artifact for academic, industrial and governmental use. This paper presents the foundations of the work in progress and we are working to extend our simulator to deal with the main challenges of forecasting load profiles. At one hand, the simulator will help the power sector in dealing with problems related to the grid e.g., evaluating network overloading, minimizing associated blackouts and alleviating power thefts; It serves as a tool to evaluate the effectiveness of optimization techniques, in demand response and micro-grids to solve the aforementioned issues of the power sector. The utility of this tool becomes highly demanding due to the unavailability of smart meters and the means to accurately measure consumption, in developing countries. While on the other hand, our contributions directly address problems related to complex system modeling e.g., multi-scale issues, heterogeneous model coupling and model validation. They further ignite impetus to explore solutions for high performance, scalability, distributed simulations.

The rest of the paper is organized as follows: Section 2 presents a review of the related work. Section 3 describe the proposed framework. Section 4 describes the simulation procedure. Section 5 presents the analysis and discussion of the results. Section 6 provides a comparison of our framework with the state of the art. Section 7 provides conclusions and future work.

\section{Literature Review}

The research on the simulation of the household electricity demand profiles are studied in detail by many researches. However, selection of methodology greatly depends upon the purpose of the study. Grandjean, et al. (17) reviewed and analyzed twelve different domestic load curve models and categorized them into two main types: (i) Top down and (ii) Bottom up. Top down models are based on macroeconomic principles and techniques. The input data for these types of the models are generally Gross Domestic Products (GDP), current population and future population growth trends, unemployment rates and many other factors. The main purpose of these types of models is to study the energy taxes; effect of different economic scenarios on energy and environment; consequences of macroeconomic changes in the energy system; and general equilibrium effects. Bottom up models, on the other hand, compute the electricity demand from individual consumption of electric appliances installed in homes by combining factors like weather, human behavior and dwelling characteristics. Following sub-sections provide a discussion on different existing frameworks, grouped into different categories: 


\subsection{Statistical Models}

Notable contributions on the development and analyses of bottom-up models are proposed by: Paatero and Lund (7), Boßmann and Staffell (9), Kremers (10), Grandjean, et al. (17), Richardson, et al. (18), Henunis and Dekenah (19), Chuan and Ukil (20), Fisher, et al. (21), Wagner, et al. (22) and Bizzozero, et al. (23). In most of these contributions, statistical, probabilistic, empirical and Time-of-Use (ToU) approaches are used to simulate the household electricity load profiles. However, these models require complex input factors, fixed pre-defined set of appliances and are mostly limited on the number of households simulated. Furthermore, the effect of weather on usage of electric appliances is only modeled on rudimentary basis. Richardson, et al. (18) presents a model that is publicly available. Version 1 of the model simulates only one house at one time while Version 2 can simulate multiple number of houses but requires complex inputs like solar thermal system configurations, PV system configuration and building thermal model parameters. Chuan and Ukil (20) developed bottom-up modeling for Singapore by extending the model developed by Paatero and Lund (7) for $1-5$ rooms flat. However, the load curve of one day for every type of the flat is generated and then multiplied by 30 to get the average monthly consumption so that the effect of different days on electricity consumption is taken in average. Moreover, the effect of temperature on electricity consumption is not considered at all. Fisher, et al. (21) developed a bottom-up stochastic model synPRO, with high time resolution of 10 seconds for German households, however, the model is not publicly available.

\subsection{Temperature in Electricity Consumption}

There exists a strong correlation between electricity consumption, type of the day, seasonal and daily mean temperature. Ali, et al. (24) used time series technique to forecast the values of electricity consumption up to 2020 for Pakistan and confirmed that a strong correlation between extreme temperatures and electricity consumption exists. Amara, et al. (25) developed a forecasting model by combining non-parametric model and time series analysis to analyze the relationship between household electricity demand and temperature. Pruckner, et al. (3) developed a hybrid model by combining the Discrete Event and System Dynamics Simulation Methodology to predict country scale electricity demand profiles and classified it on the basis of four seasons, workdays, week days, and holidays. However, these models do not represent the household consumption by considering the electricity appliances installed in the houses and dwelling characteristics

\subsection{Data Driven Models}

With the advent of Smart Grid and Home Energy Management Systems, there are many open data sources like MIT Reference Energy Disaggregation Data Set (REDD), Electricity Consumption and Occupancy Data Set (ECO) and Dutch Residential Energy Data Set (DRED) from which circuit level energy consumption profile of household can be established. Shiraz et al. (26) used MIT REDD dataset to identify hourly power consumption patterns of different appliances. L. Stankovic et al. (27) used smart meter data to infer the domestic electricity consumption by applying the techniques of Individual Appliance Monitors (IAM), Non-Intrusive Appliance Load Monitoring (NILM) and activity ontologies using surveys and interview data. Ahmed, et al. (28) combined the NILM techniques with Artificial Neural Network (ANN) for short term household load forecasting. Coelho, et al. (29) developed a self-adaptive fuzzy model for microgrid and large grids. Similarly, Danladi et al. (30) developed a long-term forecasting model on basis of fuzzy logic, however the focus of both researchers was not the domestic use but long-term demand forecasting. Giasemidis, et al. (31) used genetic algorithm technique to model the residential consumers demand behavior on low voltage networks wherein unmonitored residential consumers are assigned the same load curve as the monitored residential consumers. Razaa and Khosravic (32) reviewed the Artificial Intelligence and Hybrid Artificial Neural Network (ANN) techniques for load forecasting in combination with fuzzy logic, expert system, genetic algorithm and support vector machines. However, these models can only forecast the total energy use and are much difficult to apply in demand response scenarios. Various simulators have been proposed to resolve this issue including US department of energy's EnergyPlus (21) to Paatero and Lund's (7) bottom-up model. However, what the authors have observed is that the models in general are either structural and environment, as is the case of EnergyPlus, or socio-anthropologic as is the case of Paatero model. One of the reasons for this divergence is due to the modeling techniques that are used. Mathematical models tend to model structure and environmental data better and probabilistic models tend to model social behavior appropriately. However, what is required is a strategy which can incorporate both the structural- 
environmental and socio-anthropological aspects within the simulation element. Though very effective, data driven models are dependent on consumer data. With the drastic increase in use of smart meters and advances in the NILM techniques, gathering this data is possible wherever the relevant technology and the data is available. However, smart meters are not universally available. In regions where smart meters are not available or their data is not provided due to legal or privacy concerns then the application of the aforementioned techniques will be difficult to implement. In comparison the proposed model can be generated from different sources and stereotypical behavior of different socio-demographic households.

\subsection{Agent Based Models}

Gonzalo, et al. (33) used agent-based simulation approach by dividing the London urban area into zones using socio demographic parameters. For each zone a heterogenous group of agents are generated with occupancy profile to simulate the hourly electricity consumption for heat-pumps, electric vehicles and residential energy. The focus of Gonzalo, et al. model was electric vehicles and the right residential use was represented as an aggregate in total electricity consumption. M. Zheng, et al. (34) developed an agent-based stochastic appliance level demand model to randomly generate demand profiles for a single representative household of the U.S and then developed dispatch strategies according to available demand response tariffs to evaluate the economic viability of various available storage technologies using a simulation-based approach. Wang, et al. (35) proposed a multiagent system framework for study of household electricity consumption under varying price schemes. The case study discussed simulations of a single household with the single type of occupancy pattern under four demand response strategies. Elie, et al. (36) proposed strategies for the sustainable building performance by representing the occupants of building as agents and their thermal comfort. The focus of the proposed model is the integration of human activity with building performance. Zhang, et al. (37) used an agent-based model to study the electricity consumption in an office building. Lin, et al. (38) proposed an agent-based model to study the office building electricity consumption under a tiered price mechanism. Haiyang, et al. (39) used multi-agent system modeling and simulation to study electric vehicle penetration rates and their charging patterns to investigate smart charging strategies. Philipp et al. (15) reviewed the use of agent-based modeling and simulation approach in context of the smart electricity grids and markets.

\subsection{Models with Occupancy Patterns}

The other important factor of household electricity consumption is the occupancy patterns of the households. The households with no children or where working couple lives consumes less electricity than a household with children and older people living at home. Different occupant behavior models were reviewed on the basis of size, resolution and complexity (40). Household occupancy and activity patterns using non-homogeneous Markovchains were introduced by Richardson, et el. (18) and Widén and Wäckelgård (41). Aerts, et al. (42) developed a probabilistic three-state occupancy model at 10 mins resolution which consists of absent, at home and awake or asleep states. Similarly, Mckenna, et el. (43) developed a four-state occupancy model which consists of occupancy state ( $1=$ "at home" and $0=$ not at home") and activity state ( $1=$ " active", $0=$ "not active"). Flett and Kelly et al. (44) developed a domestic occupancy model at a one minute resolution, and introduced multiple occupant behaviors, type of day (weekday or weekend), related adults as single entity and higher order markov-chains, for the duration of the activity. Marshall, et el. (45) used three different occupant classes i.e. Working Family, Working Couple and Daytime-present couple to represent the occupancy patterns in UK Households. University of Southampton (UoS) (46) reviewed 52 documents on the occupancy patterns, out of which 16 studies were related to the UK; domestic occupancy patterns identified in these studies were Short Occupancy A, Short Occupancy B, Partial Occupancy, Home Stay A, Home Stay B.

\subsection{Model Validation}

In the domain of electricity consumption different researches have proposed different measures to validate the simulated results with the actual load profiles. Fisher et al. (47) used correlation analysis and mean relative error. The former refers to a method of statistical evaluation to study the strength of a relationship between two numerically measured, continuous variables, while the latter deals with the validation technique, where for each entry a Mean Relative Error (MRE) is computed to calculate the goodness of fit. Shiraki, et al. (48) used Root Mean Square Error (RMSE) and Mean Absolute Error (MAE). RMSE is calculated by (i) squaring the residuals, 
which is the difference between the actual values and the simulated values; (ii) averaging the squares, and (iii) taking the square root. MAE is the average horizontal or vertical distance between two continuous variables of paired observations. Javed, et al. (4) used the three measures in validation: precision, accuracy and stability. Precision is the measure of closeness of the forecasts with the actual load. Accuracy is the measure of how many correct forecasts are made. Hong, et al. (49) and Glasgo, et al. (50) referred different standards such as Federal Energy Management Program (FEMP), International Performance Measurement and Verification Protocol (IPMVP) and American Society of Heating, Refrigerating and Air-Conditioning Engineers (ASHRAE) used worldwide. Glasgo, et al. used ASHRAE Guideline 14 for Co-efficient of Variance of the Root Mean Square Error $\mathrm{CV}(\mathrm{RMSE})$ and relative annual error as reference value for comparison of Energy Plus Simulation and Actual Pecan Street Home Data collected using energy audit and home owner survey records. As per ASHRAE Guideline 14; a model is considered calibrated if hourly CV(RMSE) is less than $30 \%$ and monthly CV(RMSE) is less than $10 \%$.

\subsection{Demand Response and Microgrids}

Dehkordi et al., (51) propose a distributed noise resilient secondary control to regulate voltage and frequency of an islanded inverter-interfaced microgrid that includes several distributed generation units. Afshari et al., (52) presents a fault-tolerant control algorithm for the voltage and frequency regulation of autonomous microgrids with different faulty conditions. Such conditions include time-varying bias faults, under the switching of unreliable networks. In addition to these disturbances, Baghaee et al., (53), (54), (55), (56) propose methods to regulate some larger signal disturbances caused by short circuits, line outages, heavy motor startings etc. using a fuzzy logic controller. Kumar et al., (57) present the energy efficiency evaluation of a commercial building with strategic energy auditing and demand-side management (DSM) under different environmental conditions. Baghaee et al., (58) propose an improved decentralized hierarchical control scheme to provide nonlinear power sharing and voltage harmonic compensation of multi-DER (distributed energy resources) microgrids with nonlinear and sensitive loads. Baghaee et al., (59), (60), (61) propose different solutions to improve signal stability and powersharing of hybrid AC/DC microgrids. These contributions and the references therein provide a broad class of control problems in power systems.

The simulator proposed in this paper not only provides a platform to simulate power consumption at house and community levels, however, it also provides basis to evaluate different control strategies where consumption is not empiricaly measured. The control strategies are used to secure power system, avoid network overloading, minimize generation - consumption gaps etc. The strategies are formulated in demand response and microgrid frameworks, in the literature, to achieve the said objectives. The following account gives an overview of different control problems which can be evaluated / implemented (even in the communities which lack smart meters) with the help of the proposed simulator.

\subsection{Comparison with the State of the Art}

In this section we present a feature wise comparison of different existing $A B M$ and non-ABM frameworks, we also compare them with our proposed framework as shown in Table 1. Key parameters selected for the comparison rubric are described as follows:

- Model Resolution: It specifies the level of details covered by the model. We divide the resolution into three categories: Macro, Meso or Micro level.

- Model Scale: It specifies the size of the data e.g., no. of entities covered.

- Hierarchical: It specifies whether the model is monolithic or hierarchical. If it is hierarchical, to what extent.

- Forecast range: It specifies the horizon of the model i.e., the short term, medium, or long-term forecast.

- Exogenous Variables: This criterion suggests if the model is sensitive to external variables, if so which ones. 
Table 1: Feature wise comparison of different existing ABM and non-ABM frameworks

\begin{tabular}{|c|c|c|c|c|c|c|c|c|}
\hline \multirow{2}{*}{$\begin{array}{c}\text { Model } \\
\text { Type }\end{array}$} & \multirow{2}{*}{ Source } & \multicolumn{3}{|c|}{ Model Resolution } & \multirow{2}{*}{ Model Scale } & \multirow{2}{*}{ Hierarchical } & \multirow{2}{*}{$\begin{array}{c}\text { Forecast } \\
\text { Range }\end{array}$} & \multirow{2}{*}{$\begin{array}{c}\text { Exogenous } \\
\text { Variables }\end{array}$} \\
\hline & & $\begin{array}{c}\text { Macro } \\
\text { Level }\end{array}$ & $\begin{array}{l}\text { Meso } \\
\text { Level }\end{array}$ & $\begin{array}{l}\text { Micro } \\
\text { Level }\end{array}$ & & & & \\
\hline \multirow{11}{*}{$\begin{array}{l}\text { Non- } \\
\text { ABM }\end{array}$} & $\begin{array}{l}\text { Paatero and } \\
\text { Lund (7) }\end{array}$ & 冈 & 冈 & $\nabla$ & $\begin{array}{l}\text { Large } \\
10,000 \text { households }\end{array}$ & $\begin{array}{l}\text { Two -level } \\
\text { Houses- } \\
\text { Appliances }\end{array}$ & $\begin{array}{l}\text { Medium } \\
\text { Term }\end{array}$ & $\begin{array}{l}\text { Social factors, } \\
\text { Weather }\end{array}$ \\
\hline & $\begin{array}{l}\text { I. Richardson et } \\
\text { al (18) }\end{array}$ & 冈 & $\square$ & $\nabla$ & Configurable & $\begin{array}{l}\text { Two -level } \\
\text { Houses- } \\
\text { Appliances }\end{array}$ & Medium Term & 冈 \\
\hline & $\begin{array}{c}\text { Chuan and Ukil } \\
\text { (20) }\end{array}$ & 冈 & 田 & $\nabla$ & $\begin{array}{l}\text { Medium } \\
323 \text { houses }\end{array}$ & $\begin{array}{l}\text { Three -level } \\
\text { Houses- Type of } \\
\text { Houses - } \\
\text { Appliances }\end{array}$ & Medium Term & 冈 \\
\hline & Fischer (47) & 冈 & $\nabla$ & $\nabla$ & $\begin{array}{l}\text { Small } \\
100\end{array}$ & $\begin{array}{l}\text { Three -level } \\
\text { Houses- Type of } \\
\text { Houses - } \\
\text { Appliances }\end{array}$ & Medium Term & Seasonal Patterns \\
\hline & Wagner (22) & 冈 & $\nabla$ & $\nabla$ & $\begin{array}{l}\text { Large } \\
10,000 \text { households }\end{array}$ & $\begin{array}{l}\text { Two -level } \\
\text { Houses- } \\
\text { Appliances }\end{array}$ & Medium Term & $\begin{array}{l}\text { Season, Type of } \\
\text { the day: } \\
\text { Weekend/Week } \\
\text { Day }\end{array}$ \\
\hline & Bizzozero (23) & 冈 & $\nabla$ & $\nabla$ & $\begin{array}{l}\text { Medium } \\
200\end{array}$ & $\begin{array}{l}\text { Two -level } \\
\text { Houses- } \\
\text { Appliances }\end{array}$ & Medium Term & $\begin{array}{l}\text { Season, Type of } \\
\text { the day: } \\
\text { Weekend/Week } \\
\text { Day }\end{array}$ \\
\hline & $\begin{array}{c}\text { Ahmed. F. } \\
\text { Ebrahim (28) }\end{array}$ & 冈 & 冈 & $\nabla$ & $\begin{array}{l}\text { Small } \\
1 \text { Household }\end{array}$ & 冈 & Short Term & 冈 \\
\hline & Danladi Ali (30) & $\nabla$ & 冈 & 冈 & Not applicable & 冈 & Medium Term & $\begin{array}{l}\text { Temperature and } \\
\text { Humidity }\end{array}$ \\
\hline & $\begin{array}{c}\text { Georgios } \\
\text { Giasemidis (31) }\end{array}$ & 冈 & $\square$ & 凶 & Small & 冈 & Medium Term & 冈 \\
\hline & $\begin{array}{l}\text { Pruckner et. al } \\
\text { (3) }\end{array}$ & $\nabla$ & 冈 & 凶 & Country Scale & 冈 & Long Term & $\begin{array}{l}\text { Weather, Type of } \\
\text { the day: } \\
\text { Weekend/Week } \\
\text { Day }\end{array}$ \\
\hline & $\begin{array}{l}\text { Vitor N. Coelho } \\
\text { (29) }\end{array}$ & $\nabla$ & 冈 & 冈 & Not applicable & 凶 & Short Term & Temperature \\
\hline \multirow{7}{*}{$\mathrm{ABM}$} & $\begin{array}{l}\text { Bustos Turu } \\
\quad \text { (33) }\end{array}$ & $\nabla$ & $\nabla$ & 冈 & Large & $\nabla$ & Short Term & $\begin{array}{l}\text { Indoor and } \\
\text { Outdoor } \\
\text { Temperature }\end{array}$ \\
\hline & Zheng et al (34) & 冈 & 冈 & $\nabla$ & $\begin{array}{l}\text { Small } \\
1 \text { Household }\end{array}$ & V & Medium Term & Season \\
\hline & Wang et al (35) & 冈 & 冈 & $\nabla$ & $\begin{array}{l}\text { Small } \\
1 \text { Household }\end{array}$ & $\begin{array}{l}\text { Two -level } \\
\text { Houses- } \\
\text { Appliances }\end{array}$ & Short Term & $\begin{array}{l}\text { Weather and } \\
\text { Building Physical } \\
\text { Structure }\end{array}$ \\
\hline & $\begin{array}{c}\text { Elie Azar et. al } \\
(36)\end{array}$ & $\nabla$ & $\square$ & 凶 & $\begin{array}{l}\text { Medium } \\
500 \text { Occupants }\end{array}$ & $\begin{array}{l}\text { Two -level } \\
\text { Buildings- } \\
\text { Occupants } \\
\end{array}$ & Short Term & $\begin{array}{l}\text { Temperature and } \\
\text { Building } \\
\text { Structure }\end{array}$ \\
\hline & Tao Zhang (37) & 冈 & $\nabla$ & $\nabla$ & Small & $\begin{array}{l}\text { Two -level } \\
\text { Building- } \\
\text { Occupants }\end{array}$ & Short Term & 冈 \\
\hline & $\begin{array}{l}\text { Haiyang Lin } \\
\text { (39) }\end{array}$ & 凶 & 凶 & $\nabla$ & Small & $\begin{array}{l}\square \\
\text { Two -level } \\
\text { Building- } \\
\text { Occupants }\end{array}$ & Short Term & 冈 \\
\hline & $\begin{array}{c}\text { Our Proposed } \\
\text { Work }\end{array}$ & 口 & ஏ & 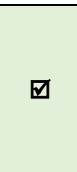 & $\begin{array}{l}\text { Medium } \\
246 \text { Households; } \\
\text { Scalable to large } \\
\text { population }\end{array}$ & $\begin{array}{l}\text { Three levels: } \\
\text { Neighborhood, } \\
\text { House, } \\
\text { Occupants, } \\
\text { Appliances }\end{array}$ & Configurable & $\begin{array}{l}\text { Hourly } \\
\text { Temperature, } \\
\text { Season, Type of } \\
\text { the day: } \\
\text { Weekend/Week } \\
\text { Day }\end{array}$ \\
\hline
\end{tabular}

It is evident from the above comparison that none of the existing frameworks support multi-resolution modeling at Macro, Meso and Micro levels, altogether. Our propose framework offers multiple resolutions, simultaneously i.e., it supports aggregate level modeling of the neighborhoods showing houses as a population of agents; (b) Group level abstraction of occupants' behaviour in each house at meso level where the individuals of a house use a common occupancy profile and exhibit the consumption behaviour accordingly; (c) Individual behaviour of electricity appliances at micro-level where different appliances in each house behave independently using state-charts. 
Our modeling approach is partially inspired from the works (4) and (20). However, our simulator implementation is completely done from the scratch using Anylogic platform. Our choice of using Anylogic Simulation software is unique, because is supports multi-method hybrid modeling. Our proposed framework is one of the few frameworks in the list that support large scale population. Scalability however is subjected to the computing power and the execution environment. In future we plan to transform our implementation into a cloud based distributed simulation platform for support a very large-scale population i.e., up to the country scale. In terms of hierarchy our proposed framework provides a multi-level Hierarchical abstraction of the real system including: neighborhood, houses, occupants and appliances. This Hierarchical distribution helps in gaining the insight of the system by applying grouped behavioural patterns. Our proposed framework provides a configurable simulation horizon. We currently use a forecast range of 1 year. Our framework can easily be interfaced with a number of external exogenous variables, to study their sensitivity in the consumption behaviour.

\section{Proposed Framework}

In this section we discuss our proposed Agent-based Modeling and Simulation framework. It is based on the foundational work on the key characteristics for assessing energy usage proposed by Arshad, et el. Our framework is designed using three-layer architecture: (i) Neighborhood layer; (ii) Social Layer and (iii) Appliances Layer. Each of these layers are connected in a hierarchical fashion as shown in Figure 2.

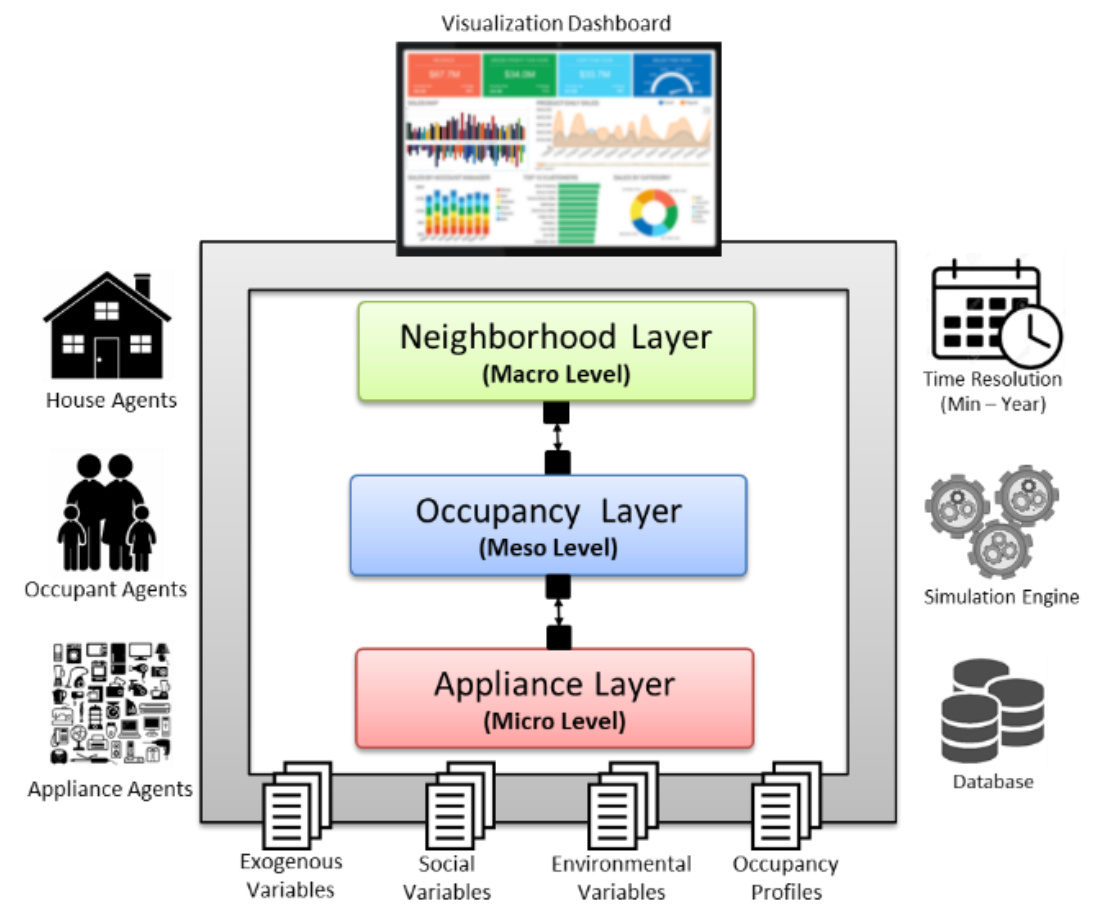

Figure 2: Hierarchical Multi-Resolution Agent Based Modeling and Simulation Framework

The neighborhood layer populates a collection of 'house agents' with parameters such as: Geo-location, number of houses, types of houses (e.g., villas or apartments), areas, size, number of beds. This population of houses forms a residential area that dynamically consume electricity overtime. It interacts with environment e.g., weather and external modules e.g., Transmission Grid and Power Supply modules.

The social layer (or Occupancy Layer) populates 'Occupant Agents', which are a group of individuals in a house, with sociological factors such as age groups, family types and living style, occupancy patterns and energy consumption behaviour. The agents in this layer interacts with the upper (neighborhood) layer for inputs e.g., temperature and with lower (appliance) layer for outputs e.g., triggers to switch on/off a device, while it provides a central role in computing the occupancy of individuals overtime.

The appliance layer populates ‘Appliance Agents', with parameters: device types, device usage, power rating and their dependence with respect to the weather. This layer models the appliance behaviour at microscopic level, and interacts with upper layers for simulating steady or intermittent load overtime. The interactions, the flow of cause-effect and the feedbacks are illustrated in Figure 3. These interactions are modeled using input parameters, state-charts, table functions, stochastic probabilities and logical reasoning. The emergence of all the households 
in use, their electrical appliances and their combination with the exogenous variables like weather, seasons and sociological factors provide estimations for the energy consumption of a typical urban neighborhood at a selected time resolution, ranging from minutes to years. The proposed framework generates the load curve for a given number of houses on a 24 hours scale, for a period of a year so that the seasonal variations can also be taken into account.

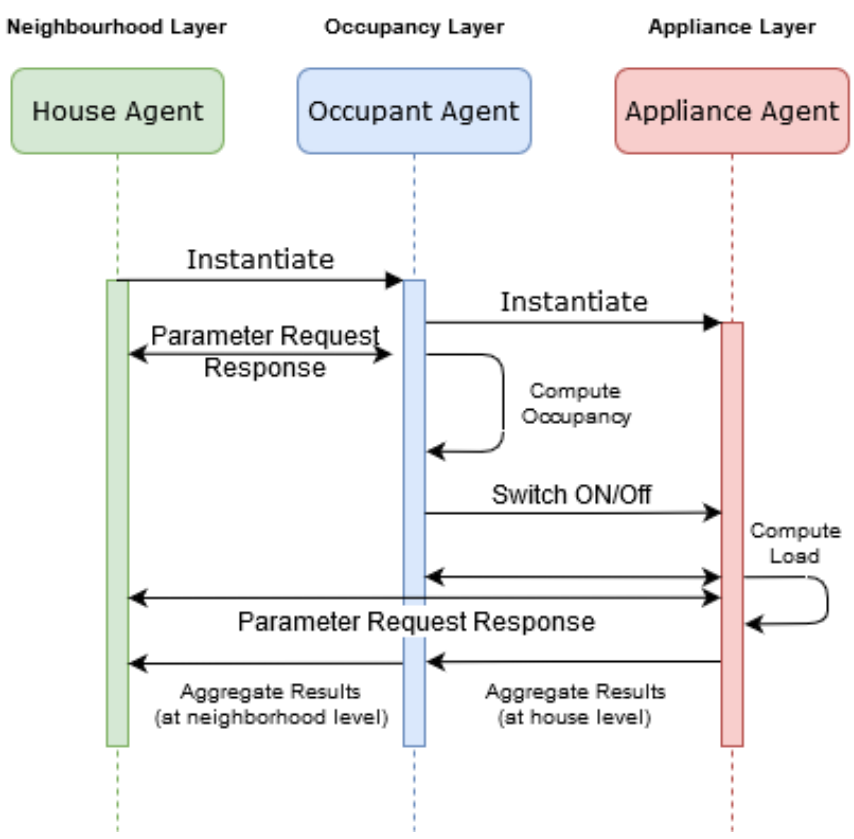

Figure 3 Agent Interaction Diagram
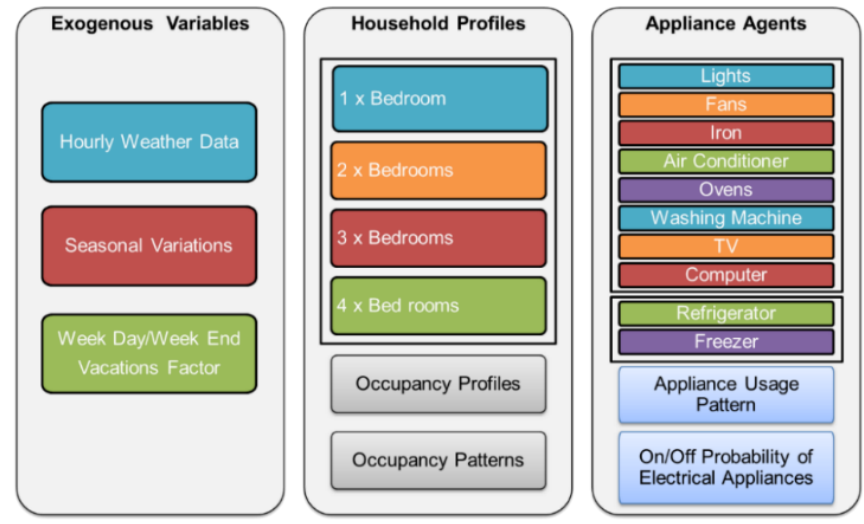

Figure 4: Elements of the proposed framework

Our framework is hierarchical, multi-resolution, multi-scale, dynamic, agent-based implementation. It is hierarchical because it is divided into three layers as shown in Figure 2, where agents in different layers are connected with each layer in a hierarchical fashion. It is multi-resolution because it allows the users to model, analyze and forecast the electricity consumption of domestic sector at: (a) macro-level, (b) meso-level and (c) micro-level, at the same time. At macro-level it allows modeling of a scalable population of households at local, regional or country scale. At meso-level it allows behaviour modeling of different groups of occupants and their sociological features. At micro-level it allows modeling of individual appliances and their energy consumption. A block diagram of the proposed framework is shown in Figure 4, which shows different elements and the entities of the ABM model. Our framework uses a built-in database, to import input data, thus allowing flexibility to the users to model and run the simulation on different datasets, therefore it is dynamic and can easily be used to study any type of population of any region. It uses an underlying Anylogic Simulation engine for computing the next step of the simulation, based on the time resolution selected by the user. 


\subsection{Exogenous Variables}

We initially define weather, seasonal variations and vacations factors as exogenous variables as examples to show how they affect different layers of our framework in terms of energy consumption. Due to the strong influence of the human behavior on the use of domestic electricity appliances, the consumption pattern of the household electricity consumption is observed to be very dynamic. Even two households with similar configurations will show different load curves. We divide the household electricity consumption into two types: (i) User-independent consumption or base load which constitutes the perpetual use of fridge and freezers; and (ii) User-dependent, which is related to the occupant activities like cooking, washing and cooling or heating requirements. The occupant activities are further divided into temperature dependent and temperature independent appliances. Users may incorporate more variables to incorporate demographic, econometric and sociologic parameters in order to study their influence, and therefore can benefit from our open-ended implementation.

\subsection{Household Profiles}

Household electricity consumption is influenced by socio-economic indicators like dwelling types, household income, appliance holdings, power ratings, number of occupants, among many others (62). Electricity consumption is also highly correlated with the area of the floor (63). Larger floor areas will have more lighting requirements and is linked with high income groups i.e., the ones with more ownership of the electrical appliances. In our proposed framework, the houses are divided into four types as listed in Table 2. The profiles are used in the initial configuration of the house-agent population. The model can be extended for any number of bedrooms and with any income groups.

Table 2: House Profiles

\begin{tabular}{cccc}
\hline No & House Profile & No. of Bed & Area $(\mathbf{s q f t})$ \\
\hline 1. & House Profile 1 & 1 & $550-750$ \\
2. & House Profile 2 & 2 & $950-1700$ \\
3. & House Profile 3 & 3 & 2700 \\
4. & House Profile 4 & 4 & 3549 \\
\hline
\end{tabular}

\subsection{Occupancy Patterns}

The occupancy patterns include the number of people residing in the household, their age groups, gender, vocation, and the probability of their occupancy within the house during the 24 hours period. In proposed model we implemented a two-state occupancy i.e., 'at-home' and 'not at home', and propose four types of occupancy patterns listed in Table 3:

Table 3: Occupancy Patterns

\begin{tabular}{|c|c|c|}
\hline No & Occupancy Pattern & Description \\
\hline 1. & WC & $\begin{array}{l}\text { Working Couple: This pattern represents the population which remains absent } \\
\text { from the house mostly from 8:00 a.m. to } 4: 00 \text { p.m. }\end{array}$ \\
\hline 2. & WCC & $\begin{array}{l}\text { Working Couple with Children: This pattern represents the household } \\
\text { population which has lower probability of occupancy at home from 8:00 a.m. to } \\
\text { 4:00 p.m. }\end{array}$ \\
\hline 3. & HS1 & $\begin{array}{l}\text { Home Stay 1: This pattern represents the household where occupants remain } \\
\text { present in house most of the time e.g., families with school going children and } \\
\text { older people. }\end{array}$ \\
\hline 4. & HS2 & $\begin{array}{l}\text { Home Stay 2: This pattern represents the household where occupants remain } \\
\text { present in the house most of the time e.g., families with house wife, infants, } \\
\text { children and older people. Only few of the members go out for work }\end{array}$ \\
\hline
\end{tabular}




\subsection{Electrical Appliances Usage Patterns}

We consider a set of daily life electrical appliances in our proposed framework as shown in Table 4. The electricity consumption, for each electrical appliance, is calculated using a mathematical model, initially proposed by (7):

$$
\mathbf{P}_{\text {start }}\left(A, \Delta \mathbf{t}_{\text {comp }}, \mathbf{h}\right)=\mathbf{P}_{\text {hour }}(\mathbf{A}, \mathbf{h}) \times \mathbf{f}(\mathbf{A}, \mathbf{d}) \times \mathbf{P}_{\text {step }}\left(\Delta \mathbf{t}_{\text {comp }}\right) \times \mathbf{P}_{\text {sat }}(\mathbf{A})
$$

Equation 1: Mathematical Model

Each electrical appliance is assumed to be switched on using a starting probability $\left(\boldsymbol{P}_{\text {start }}\right)$, during the 24 hours period. In above mathematical model, $\left(\boldsymbol{P}_{\text {start }}\right)$ is calculated on basis of three variables: $\mathbf{A}$ is representing the appliance, $\Delta \boldsymbol{t}_{\text {comp }}$ is the computational timestamp, h is hour of the day. $\boldsymbol{P}_{\text {hour }}$ is the hourly probability factor of each appliance, $\boldsymbol{f}$ is the mean daily starting frequency of each appliance, $\mathbf{d}$ is the day of the week, $\boldsymbol{P}_{\text {step }}$ is the step size of the scaling factor that scales the probabilities according to $\Delta \boldsymbol{t}_{\text {comp }}, \boldsymbol{P}_{\text {sat }}(\boldsymbol{A})$ is the saturation probability of the appliances. The mathematical model was extended by Chuan and Ukil (20) for the determination of starting probability of different appliances for 1 to 4 rooms. Furthermore, the weekday and weekend starting probabilities for appliances mentioned at Sr. No 6-7 and 10 of Table 4 are interpolated. The nominal wattage rating is taken from the local sources. The average time per cycle for each appliance is based on theoretical estimations. A time cycle is the duration for which an appliance remains turned on. When the appliance cycle time is reached, it will be turned off and model starts to re-evaluate the on/off status of the appliance for the next cycle. We use nominal wattage and the time cycles for the appliances listed in Table $4(1,6-8$ and 10-11). Later in this paper, Figure 11 shows the starting probability for each hour during the day for type 3 houses. These probability tables are built based on daily life patterns obtained from the observational site, discussed later in this paper.

Table 4: Electricity Appliances with Wattage and Cycles

\begin{tabular}{clcc}
\hline No & Electrical Device & Nominal Wattage $\mathbf{( W ) / h r}$ & Time per Cycle (min) \\
\hline 1. & Lights & 25 & 30 \\
2. & Fans & 70 & 30 \\
3. & Iron & 1000 & 30 \\
4. & Refrigerator & 110 & 12 \\
5. & Freezer & 200 & 12 \\
6. & Washing Machine & 500 & 90 \\
7. & TV & $100-300$ & 90 \\
8. & Computer & 80 & 60 \\
9. & Air Conditioner & 1500 & 120 \\
10. & Oven & 1500 & 5 \\
11. & Misc. load & 1500 & 60 \\
\hline
\end{tabular}

\subsection{Weather Dependent Appliances}

In our proposed model we included temperature as an exogenous variable as it directly affects the appliance usage. In this paper, households of countries with extreme temperatures in summer, are considered, which require air conditioning and other cooling appliances for domestic needs. Similarly, it is assumed that the temperature in winter remains moderate and therefore electricity usage for heating appliances, central heating, or water heating is negligible. In many studies, a high correlation is established between electricity consumption and weather variables like temperature, humidity, rainfall and solar radiations. In our proposed framework, a simple linear function between the temperature and probability usage of fans and air conditioners is adopted from Tsuji (64) and Shiraki (48). The temperature dependent probability of appliance usage is $0 \%$ at temperature $\mathbf{T}_{\mathbf{a}}$ and $100 \%$ at Temperature $\mathbf{T}_{\mathbf{b}}$. The temperature dependent probability of fan usage reaches $0 \%$ if temperature is below $24^{\circ} \mathrm{C}$ $\left(\mathrm{T}_{\mathrm{a}}\right)$ and reaches to $100 \%$ if temperature is greater or equal to $28^{\circ} \mathrm{C}\left(\mathrm{T}_{\mathrm{b}}\right)$. The temperature dependent probability of air conditioner usages reaches $0 \%$ if temperature is below $28^{\circ} \mathrm{C}$ (Ta) and reaches to $100 \%$ if temperature is greater or equal to $46^{\circ} \mathrm{C}\left(\mathrm{T}_{\mathrm{b}}\right)$ as shown in Figure 5. The proposed model can be extended for any type of the temperature dependent appliances. A similar model can be applied for the humidity and rainfall. Furthermore, 
instead of linear function any other function can be used to describe the usage behavior of the Fans and Air Conditioners.
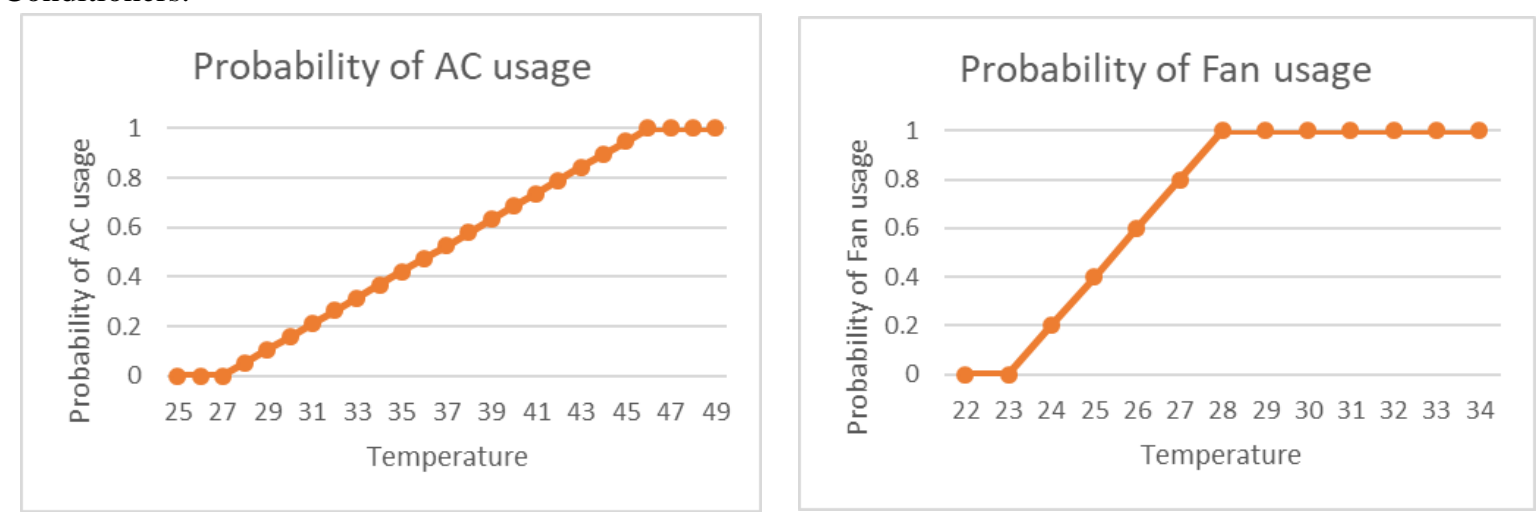

Figure 5: Temperature Dependence (a) AC Usage probability (b) Fan Usage probability

\subsection{Simulation procedure}

In this section we describe the implementation of the proposed ABM model in AnyLogic 8.2.4 University Edition, using ABM libraries. A flowchart of this procedure is shown in Figure 6. In order to compute electricity consumption per minute, we created a neighborhood agent consisting of four (4) agents representing the house types as listed in Table 2 and eleven (11) types of electrical appliance agents as listed in Table 4 . The number of the electrical appliances vary for the different types of houses. An initial population of the house agents is dynamically created. This procedure is configurable through user inputs.

The behaviour of appliance agents is encapsulated within the house agent. The house agent evaluates the occupancy over time. If the house is occupied, it may switch on or off the appliances using conditional transitions shown in Figure 12. Using the probability distributions, assigned to each appliance. All these agents are encapsulated within neighborhood agent; hence the hierarchy is formed. The neighborhood agents aggregate the domestic consumption of the houses within and is designed to interact with external modules e.g., Transmission Grid and Power distribution to study variations of domestic load, and for demand side management or demand response management.

\subsubsection{Behaviour of Neighborhood Agent}

Neighborhood agents are modeled to initialize the simulation data and instantiate household population agents. It is also used to perform energy consumption calculations. Figure 7 shows the structure of the neighborhood agent using the symbols used in Anylogic Simulation Software

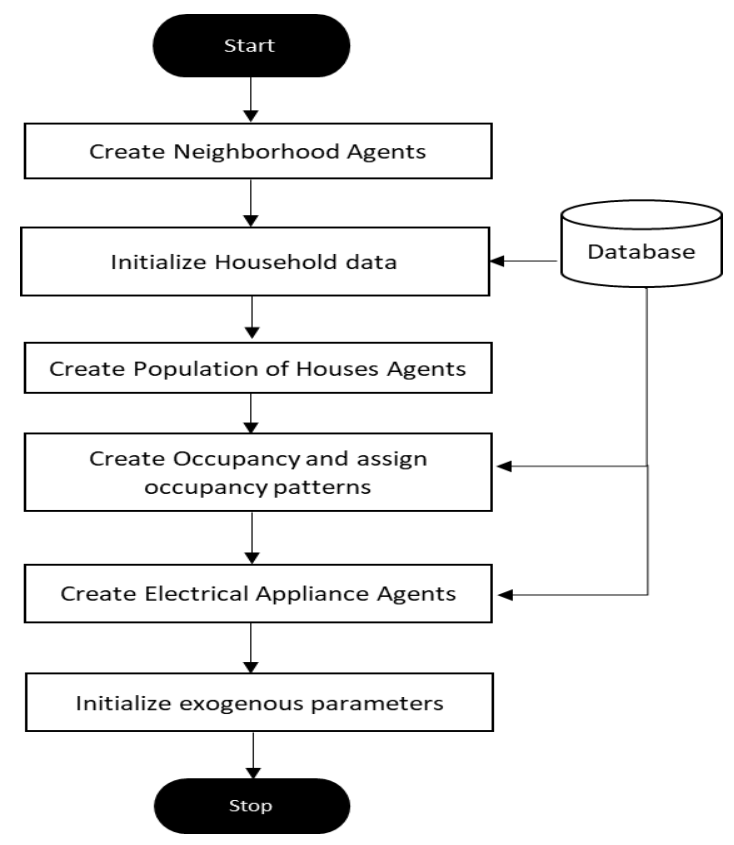

Figure 6: Flow Chart for Model Initializations 


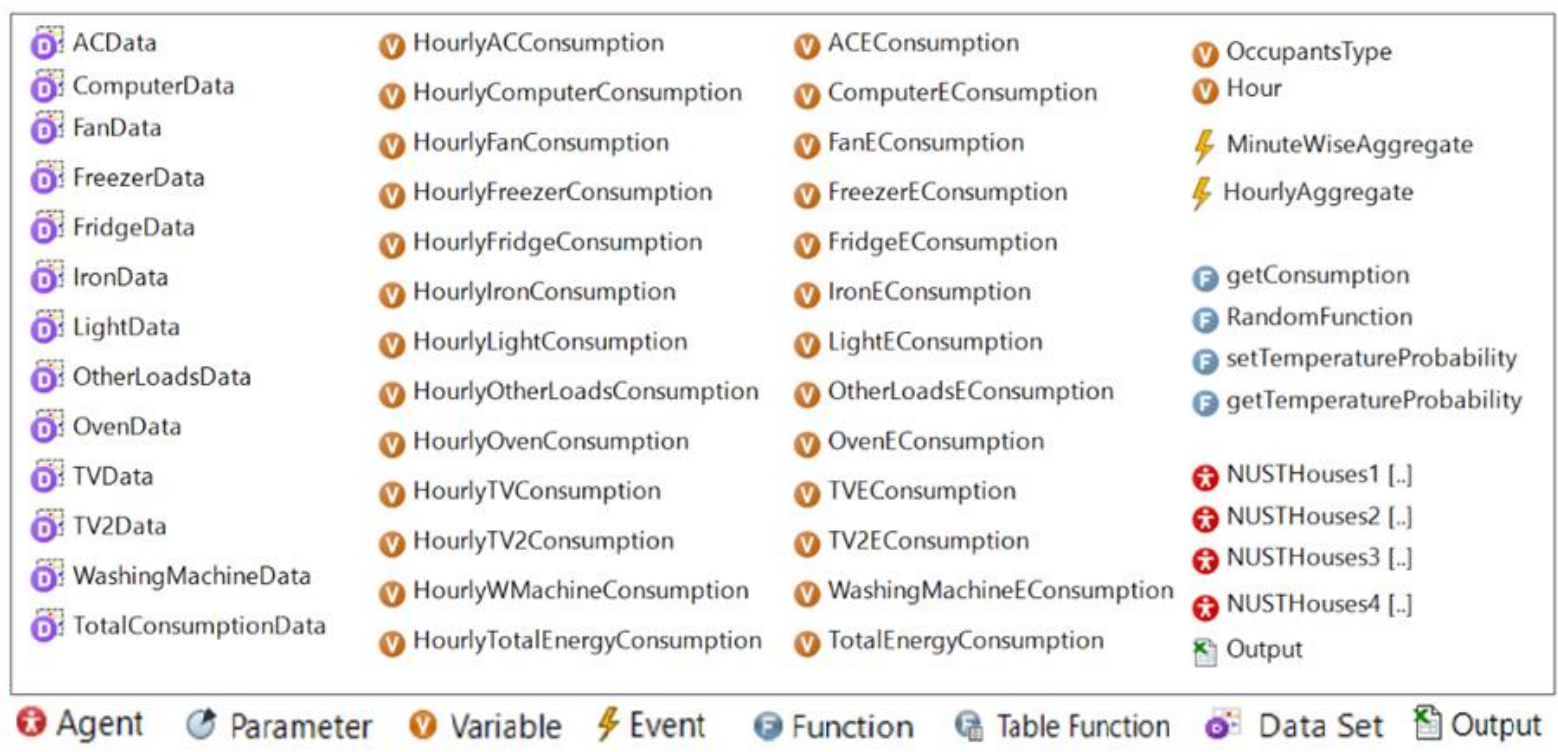

Figure 7: Neighborhood Agent
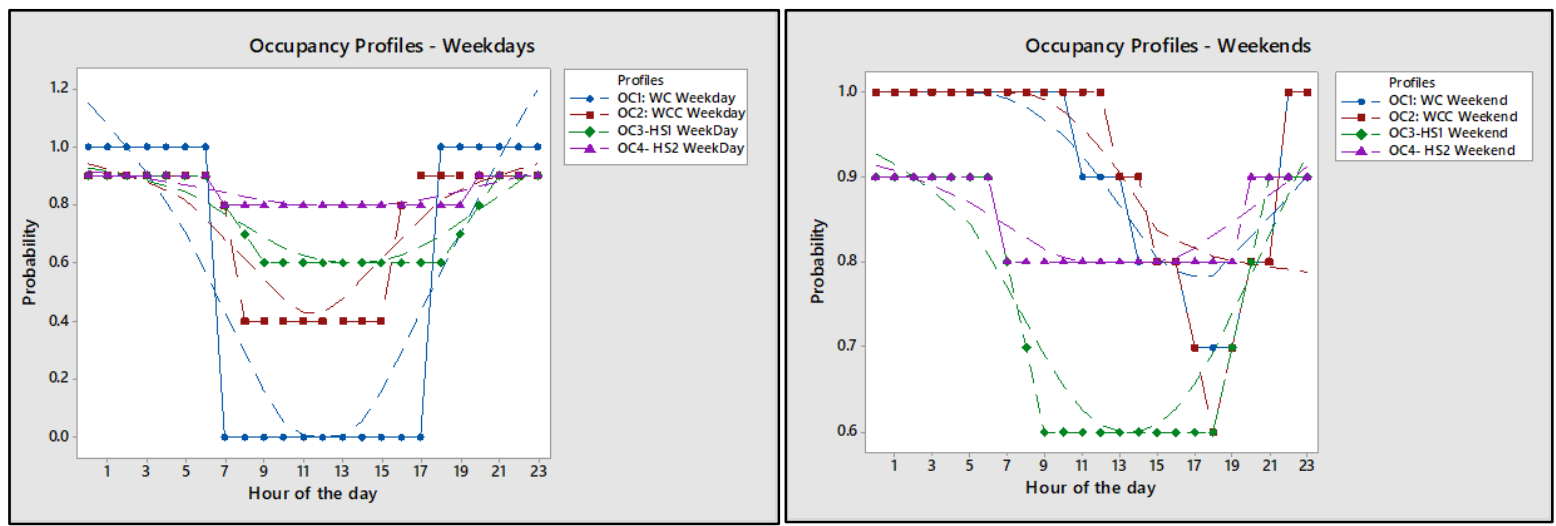

Figure 8 : Occupancy Profiles during week days and weekends

\subsubsection{Behavior of Household Agent}

A household is an agent that is instantiated within a neighborhood agent and is initialized dynamically using the input data obtained from the house table of the database. The main purpose of this agent is to manage the occupancy of its occupants using a specified occupancy profile and the occupancy state-chart as shown in Figure 9. When the simulation starts, the occupants are initialized in the available occupant variable and their number increases or decreases due to the 'recheck' transition that occurs periodically and based on the occupancy profiles, computes the new occupancy. The occupancy behavior of household's agents listed in Table 3 is implemented by using table functions shown in Figure 8. Table functions are elements from the Anylogic toolbox and are used to define complex non-linear relationships which cannot be described in standard functions. The table functions can take discrete values as input and can be made continuous by interpolation and/or extrapolation. The profiles use different variants of these table functions to distinguish between the weekdays, weekends, national holidays and seasonal vacations. In this paper we consider both weekdays and weekends. Our framework allows user inputs for adding or editing the occupancy profiles and allows dynamic analysis of the effects of different occupancy behavior on the energy consumption at runtime. In each household agent a set of appliance agents are dynamically populated using the input data obtained from the appliance table of the database. 
House Data

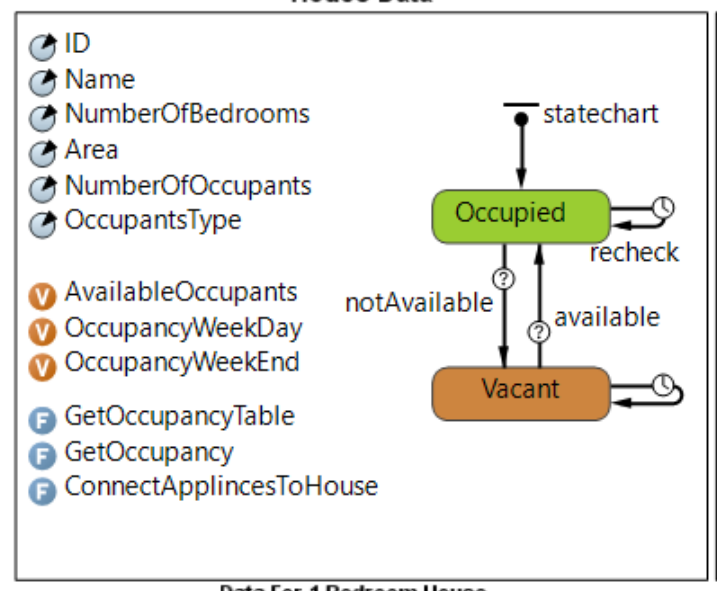

Data For 1 Bedroom House

\begin{tabular}{|c|c|}
\hline 6 P1ACSeasonalProb & P1ComputerUsageProb \\
\hline P1FanSeasonalProb & P1FreezerUsageProb \\
\hline P1FridgeUsageProb & G1 P1ronUsageProb \\
\hline$E^{\text {P1LightUsageProb }}$ & $G_{\text {P1OvenUsageProb }}$ \\
\hline P1OtherLoadUsageProb & $G^{\text {P1TVUsageProb }}$ \\
\hline P1TV2UsageProb & G1WMachineUsageProb \\
\hline
\end{tabular}

Data For 2 Bedroom House

\begin{tabular}{|c|c|}
\hline 6 P2ACSeasonalProb & P2ComputerUsageProb \\
\hline P2FanSeasonalProb & P2FreezerUsageProb \\
\hline P2FridgeUsageProb & P2IronUsageProb \\
\hline G2LightUsageProb & $\mathrm{C}_{\mathrm{B}} \mathrm{P} 2 \mathrm{OvenUsageProb}$ \\
\hline P2OtherLoadUsageProb & G2TVUsageProb \\
\hline 8 P2TV2UsageProb & G2WMachineUsageProb \\
\hline
\end{tabular}

SeasonalHourlyTemperature

\begin{tabular}{|c|c|}
\hline G HourlyTempFall & G HourlyTempSpring \\
\hline GourlyTempWinter & 8 HourlyTempSummer \\
\hline
\end{tabular}

Appliance Data

\begin{tabular}{|c|c|}
\hline$\rightarrow$ numberOfAC & ( Air_Conditioner [..] \\
\hline$\checkmark$ numberOfComputer & @ Computer [..] \\
\hline numberOfFan & ‡) Fan [..] \\
\hline numberOfFreezer & ㅈ Freezer [...] \\
\hline$\rightarrow$ numberOfFridge & (: Fridge [..] \\
\hline$\odot$ numberOflron & ㅊ Iron [..] \\
\hline numberOfLight & ㅊ Light [..] \\
\hline$\rightarrow$ numberOfOtherLoads & ( $)$ Other_Loads [..] \\
\hline$\rightarrow$ numberOfOven & đ Oven [..] \\
\hline numberOfTV & ¿ TV [..] \\
\hline numberOfTV2 & ㅈ TV2 [..] \\
\hline numberOfWashingMachine & đ Washing Machine [..] \\
\hline
\end{tabular}

Data For 3 Bedroom House

\begin{tabular}{|c|c|}
\hline G3ACSeasonalProb & G P3ComputerUsageProb \\
\hline P3FanSeasonalProb & G3FreezerUsageProb \\
\hline P3FridgeUsageProb & G3IronUsageProb \\
\hline G3LightUsageProb & G3OvenUsageProb \\
\hline P3OtherLoadUsageProb & G3TVUsageProb \\
\hline G3TV2UsageProb & G3WMachineUsage \\
\hline
\end{tabular}

Data For 4 Bedroom House

\begin{tabular}{|c|c|}
\hline G4ACSeasonalProb & G4ComputerUsageProb \\
\hline F4FanSeasonalProb & G4FreezerUsageProb \\
\hline P4FridgeUsageProb & P4IronUsageProb \\
\hline F4lightUsageProb & G4OvenUsageProb \\
\hline P4OtherLoadUsageProb & P4TVUsageProb \\
\hline G4TV2UsageProb & P4WMachineUsage \\
\hline
\end{tabular}

Occupancy

\begin{tabular}{|c|c|}
\hline GCOccupancy & GCWeekEndOccupancy \\
\hline G. WCCOccupancy & GCWCWeekEndOccupancy \\
\hline HS1Occupancy & GS1WeekEndOccupancy \\
\hline GS2Occupancy & G HS2WeekEndOccupancy \\
\hline
\end{tabular}

Figure 9: Household Agent

\subsubsection{Behavior of Electrical Appliance Agent}

The proposed simulation framework is configured at a per minute resolution and the control structure of the simulation is implemented using events. All electrical appliance agents respond to time-out triggered events in cyclic fashion. The recurrence time of the events for these appliances are set using time cycles listed in Table 4 . A flow chart for the behaviour of a device agent is shown in Figure 10. The electrical appliances agents are modeled as passive agents i.e., they possess event-driven reactive behavior. Since the appliance agents are populated under the hierarchy of different house agents, therefore, a unique behavior pattern for the use of each appliance agent is created using table the functions. For every appliance agent, a state chart is created which will indicate the on and off status and electricity consumption of the appliance according to the states. This behaviour can be extended using additional states e.g., 'stand by' in the case of TV. The transition from 'Off' to 'On' state is initially dependent on the occupancy profile of the household agent. If an occupant is present in the house, a randomly generated number using a Uniform $(0,1)$ distribution, is compared with the device usage probability at the corresponding time defined by the table function, shown in Figure 11. If the generated number falls within 
the probability usage value, the device agent will transit from 'Off' to 'On' state as shown in Figure 12. And the corresponding wattage will be added in the electricity consumption, i.e., when an agent is switched on, its power will be contributed in the total power of the house agent. The device will remain turned on until the time cycle of the agent is reached, after which the state-machine will recheck the number of occupants in the house. The higher usage probability will result in more likely chance for the device to be switched on. Due to the use of uniform distribution, it is likely that not all the agents e.g., all the lights in the house will switch on at once, at a particular time, which makes the model stochastic. The power ratings are instantiated as global constants, so that changes in the entire framework can be easily applied. The variability of the power rating is not considered and will be dealt with in the future work.

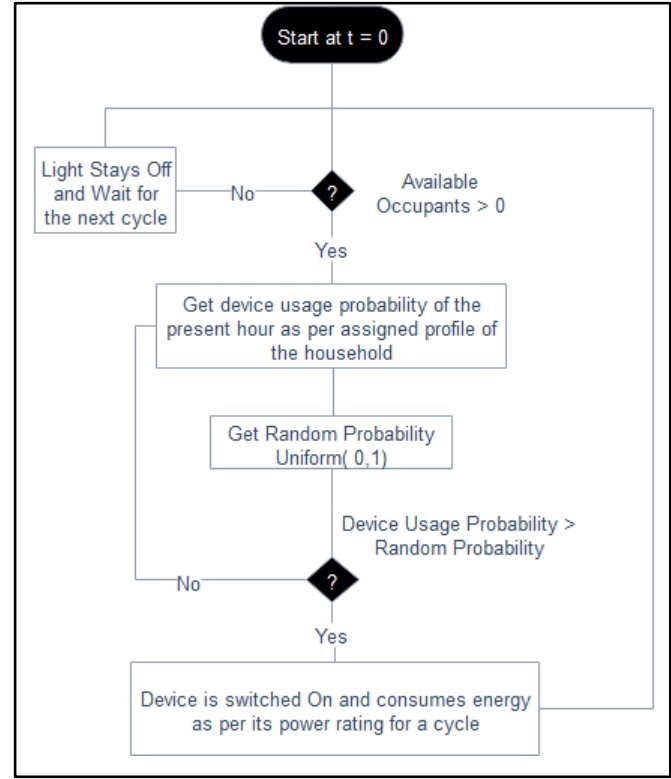

Figure 10: Behaviour of Agents

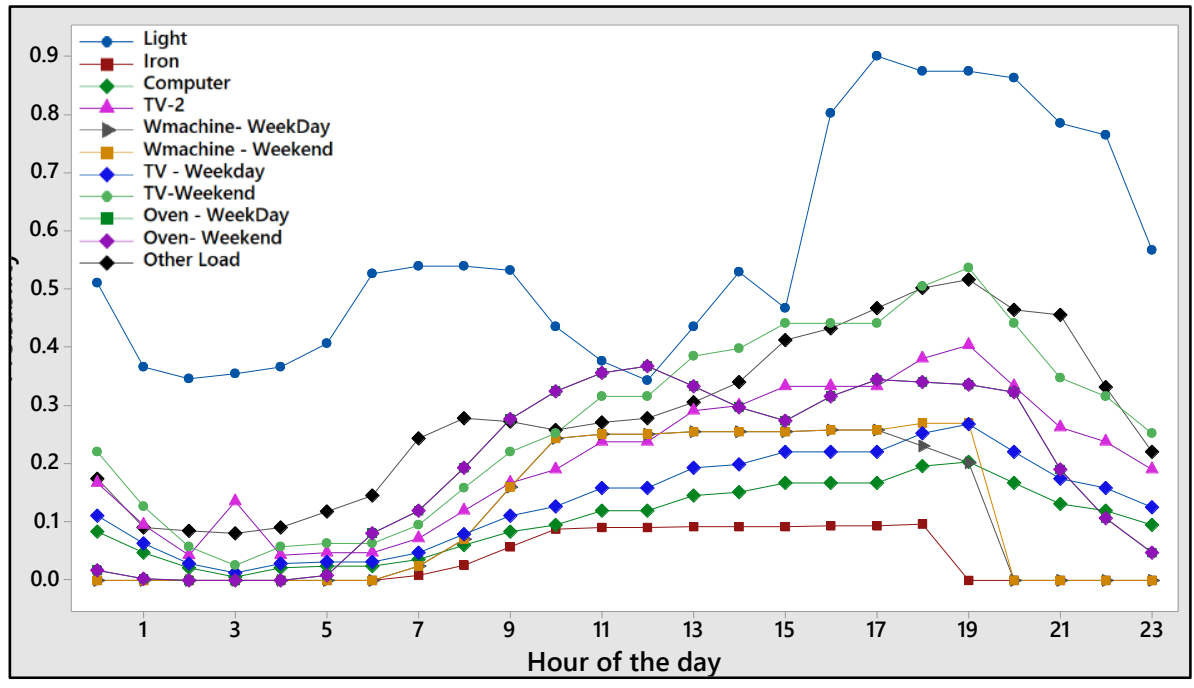

Figure 11 : Electrical Appliances Usage for Type3 House

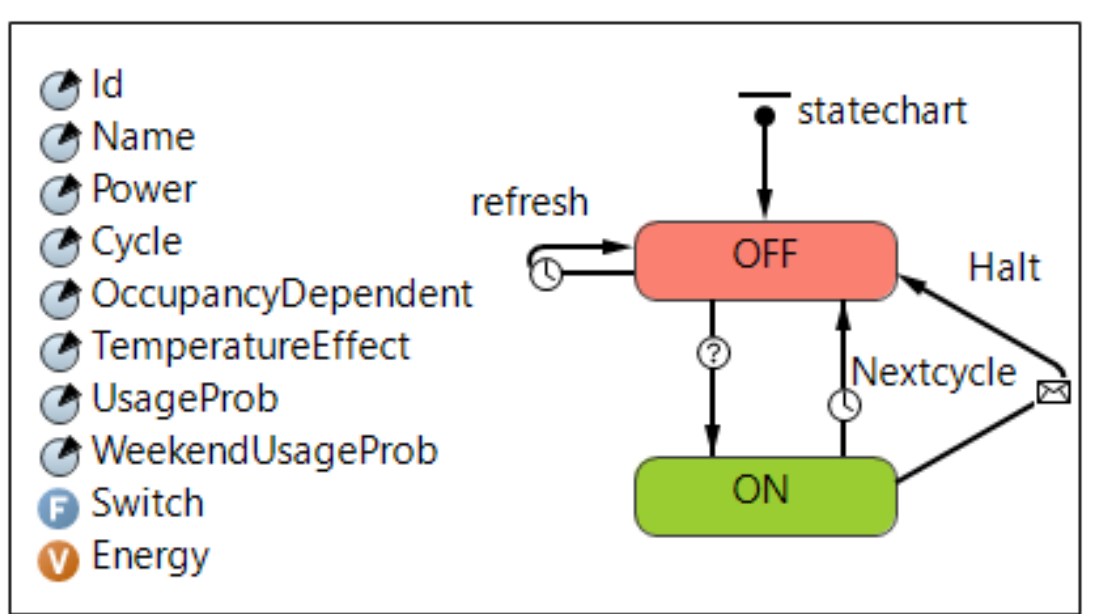

Figure 12: Appliance Agent 


\subsubsection{Electricity Consumption Calculations}

In the proposed framework, the appliance agents are classified into three types: (i) The continuous appliances that run constantly independent of the occupancy profile e.g., refrigerators and freezers; (ii) The appliances that are temperature dependent with on / off states, heavily dependent upon the rise and the fall of the temperature, along with occupancy profile, e.g., fans and air conditioners; and (iii) The appliances that are only dependent upon the occupancy profiles and type of the day i.e., weekday or weekend. The 'per minute' energy consumption for every appliance agent is calculated which is then aggregated for every house and then for all houses using the following equation:

$$
E_{A}=\sum_{i=1}^{h}\left(\sum_{j=1}^{k}\left(\sum_{t=1}^{24 h r s}\left\{\begin{array}{lr}
N_{\text {rand }}<\text { DeviceOnProb }_{t} & \text { Power }_{A} \\
\text { else } & 0
\end{array}\right)\right)\right.
$$

Equation 2: Electricity Consumption

Where $E_{A}$ is the total energy of an appliance during the 24 hours period and is the sum of the electricity consumed by that device during its 'On' state. Power $_{A}$ is the power rating used for the appliance $E_{A} . N_{\text {rand }}$ is the random number generated using uniform probability distribution $\mathrm{U}(0,1)$ and DeviceOnProb $_{t}$ is the device usage probability at the time $t$. ' $\mathrm{k}$ ' represents the total number of devices in a house and $\mathrm{h}$ represents the total number of houses used in the simulation scenario.

\section{Model Validation and Results}

In this section we present a case study conducted on the housing area of the campus of National University of Sciences and Technology (NUST), Islamabad, Pakistan. The purpose of this case study was to compare and evaluate the simulation behaviour of our proposed framework with real-world data.

\subsection{Data Collection and Analysis}

We collected actual load data from the electric supply company for 264 domestic consumers, for four different seasons and compared it with the simulation results. The actual load data is for the duration of one year from July, 2016 to June, 2017 at a resolution of fifteen-minutes. The mean season wise load is shown in Figure 13. It can be noticed that consumption in winter is minimum because the fans and ACs are completely switched off. And the thermostats of the fridge and freezers are lowered. In fall, there is a partial use of fans. In spring, there is a greater use of fans and partial use of ACs. In summer, both fans and ACs are extensively used however, the collected data does not reflect this, as expected. It was identified that most of the population goes out for vacation in summer.

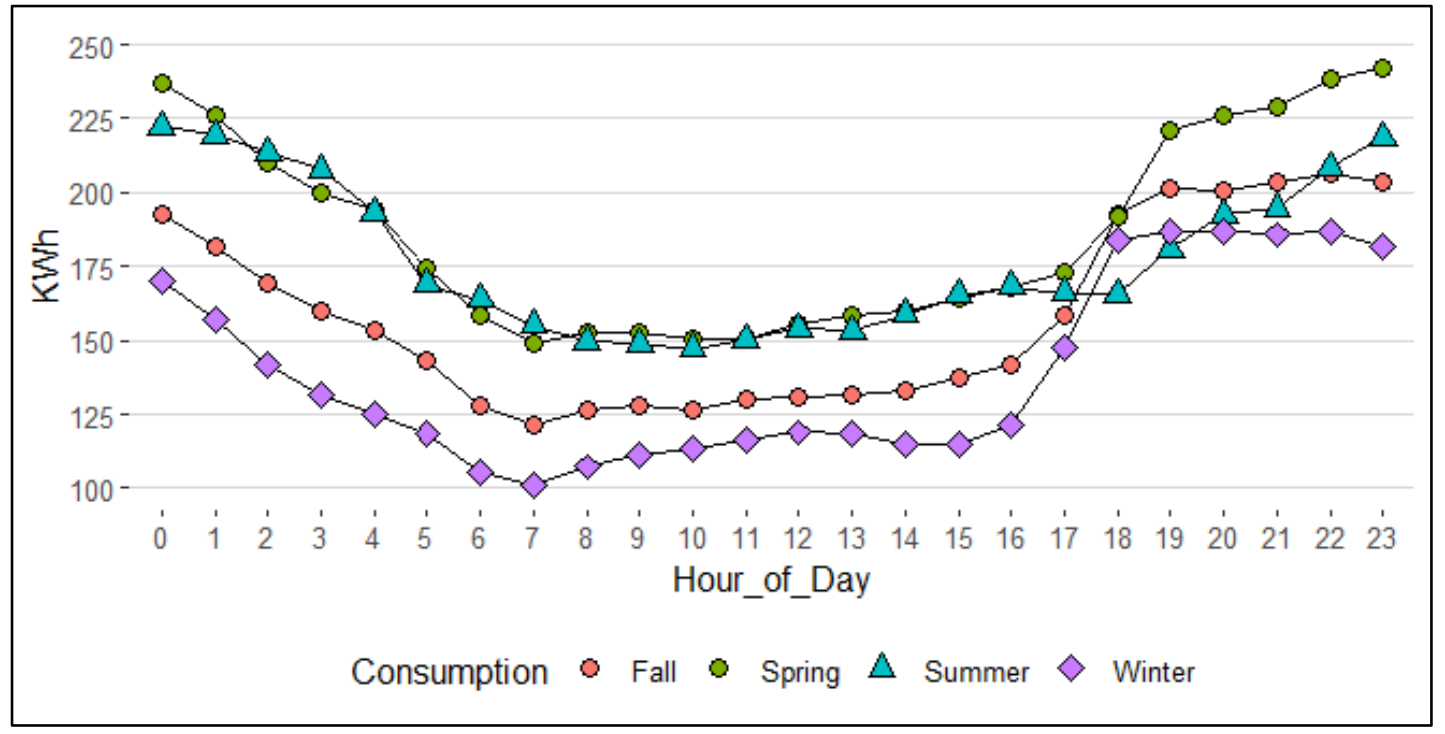

Figure 13: Average daily load for different seasons (kWh) 
The box plot of the complete year in Figure 14. is showing a clear trend that consumption at the time from 8:00 a.m. to 3:00 p.m. is lower as compared to other time. The lower consumption at these times can be clearly associated with the working residents. We also examined the correlation between temperature and electricity consumption. Figure 15 shows the scatter plot for the temperature and load in MW, showing an increase in temperature leads to more electricity consumption. The $\mathrm{p}$-value analysis with co-efficient $=0.37$ also confirms the correlation, indicating a positive linear trend.

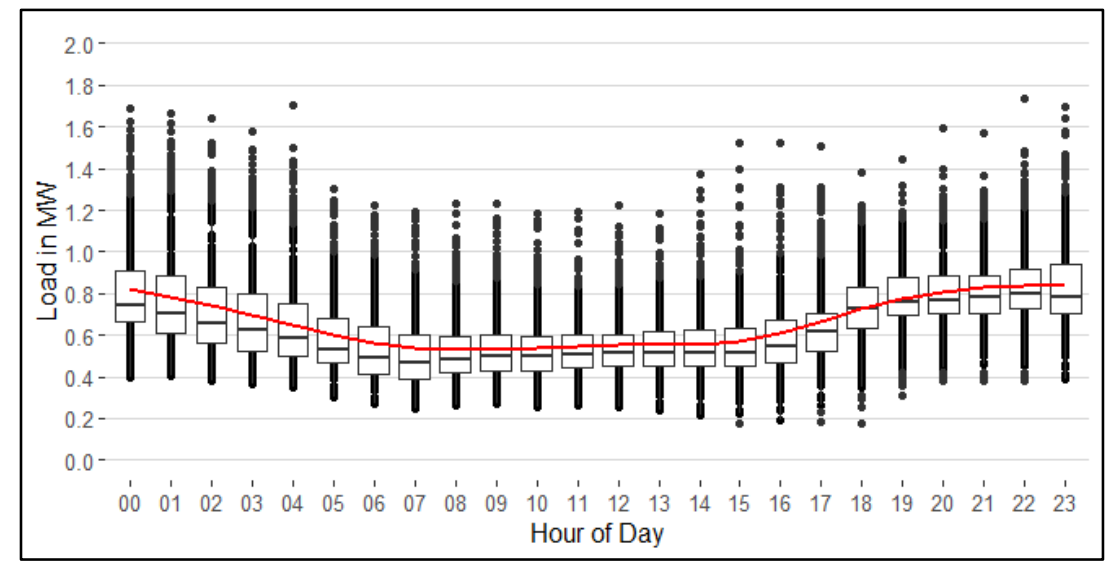

Figure 14: Box Plot of daily consumption (1-year data)

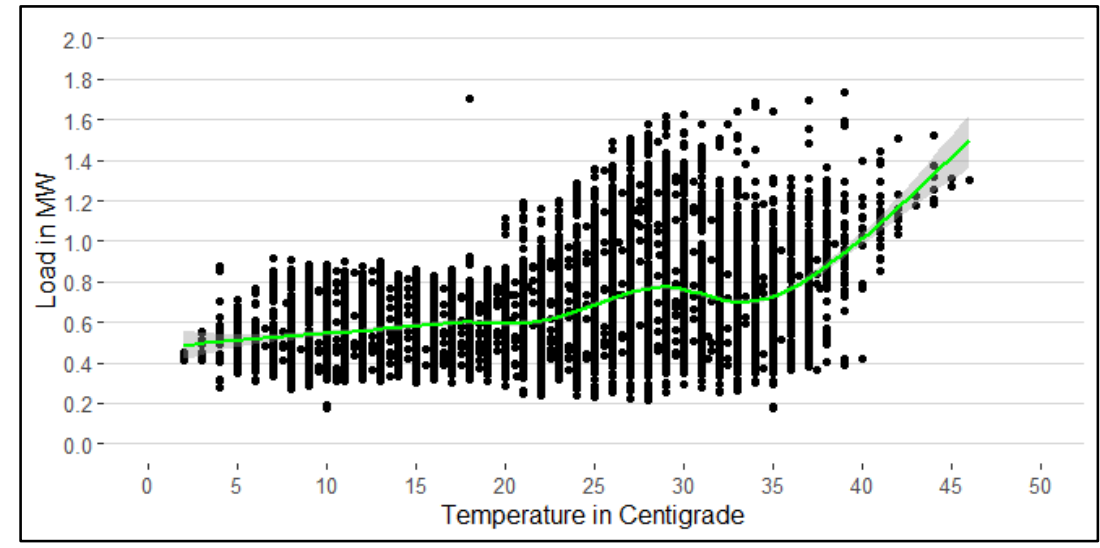

Figure 15: Temperature vs Load in MW (MWh)

\subsection{Simulation Experiment}

We ran a simulation experiment of a neighborhood of 264 houses that consists of 7 houses of four bedrooms, 51 houses of three bedrooms, 142 houses of two bedroom, and 64 houses of one bedroom. The number of houses and other parameters like number of bedrooms, number of occupants, occupancy patterns and electrical appliances present in the house are initialized in database at the start of the simulation. We ran a simulation experiment of 5 iterations for a year and the average result of the simulated consumption was compared with the actual consumption. The appliance wise hourly consumption for a day is shown in Figure 16. It can be seen that the lights, fans and fridge have prominent consumptions. The total consumption and actual consumption are represented in blue and red line respectively. Mean hourly temperature of the year is also illustrated. It can be seen from the Figure 16 that our simulator underestimates the consumption between the period from 12:00 AM to 8:00 AM in the morning, as compared to the actual consumption. Whereas it overestimates the consumption between 6:00 PM to 11:00 AM in the evening. This deviation is due to the sensitivity of the appliance usage probability tables on the model, and can be adjusted by the user by calibration.

A comparison of season wise appliance level consumption is shown in Figure 17. The season wise simulations for the whole year was run and the comparison of mean simulated load and actual load are shown in Figure 18: Season wise hourly consumption (a) Fall (b) Spring (c) Summer and (d) Winter. 


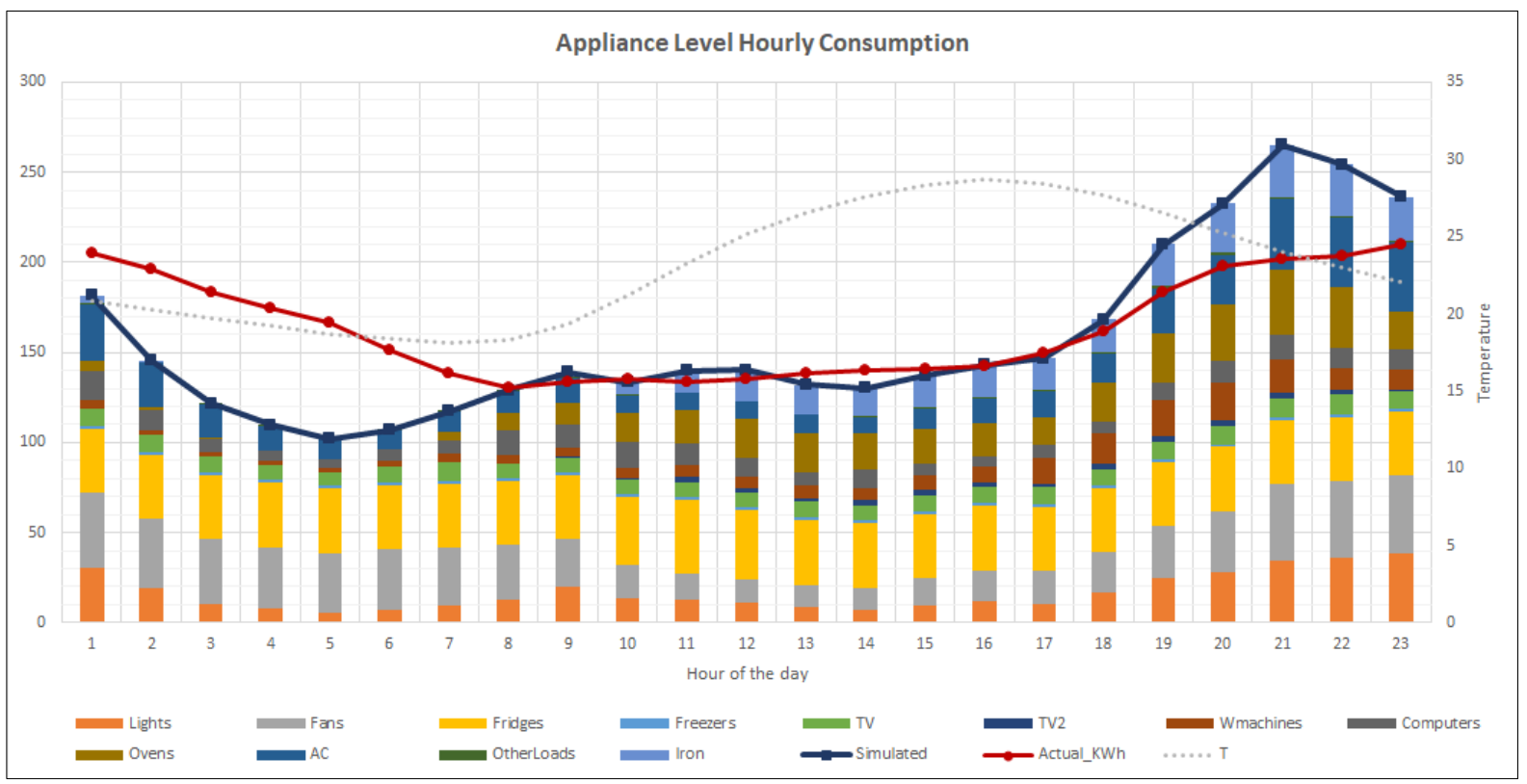

Figure 16: Appliance level Hourly Consumption of 264 Households

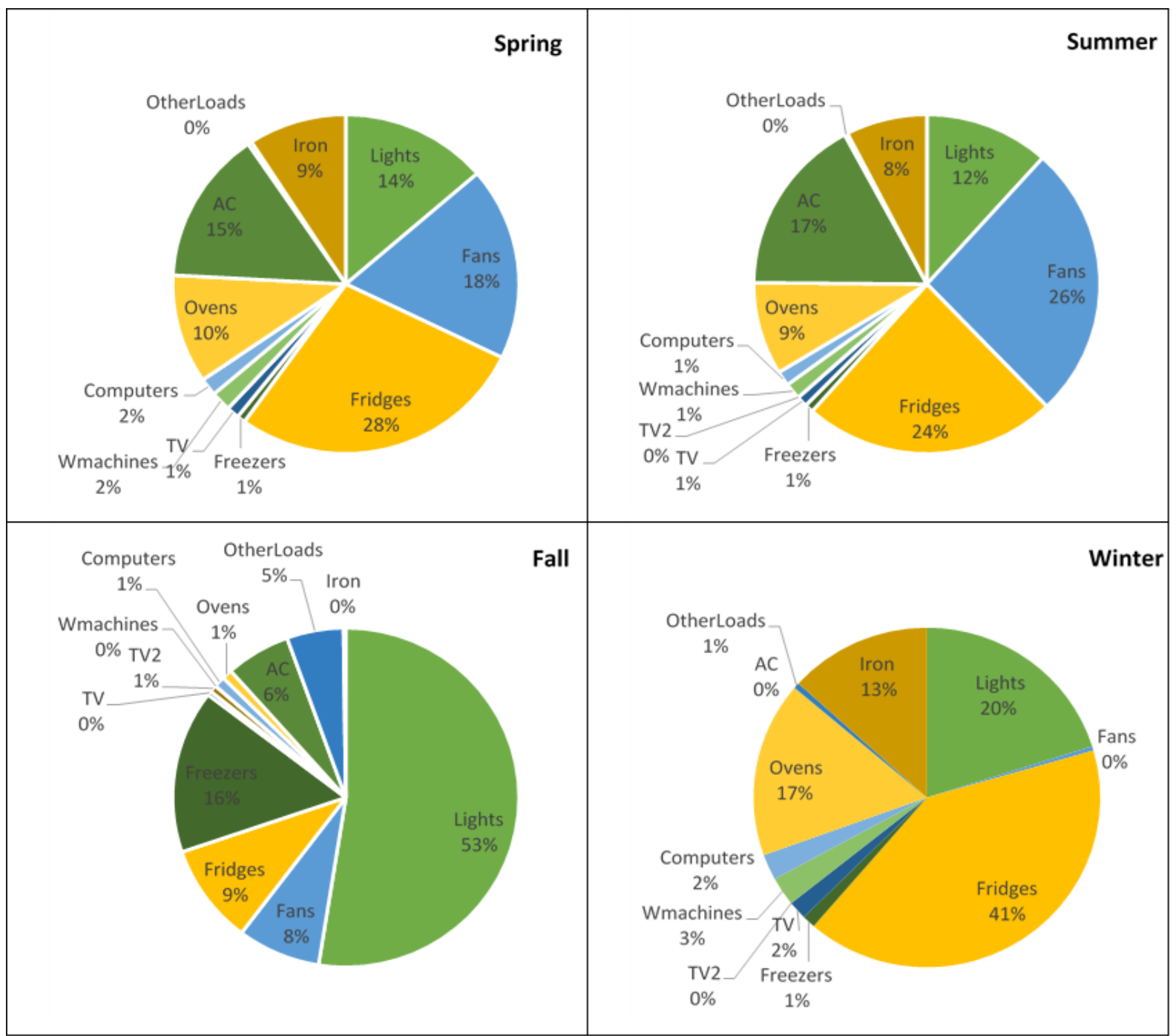

Figure 17: Season Wise Consumption at appliance level 
Seasonwise Hourly Consumption - Actual vs Simulated

$\longrightarrow$ Simulated $\longrightarrow$ Actual $\quad---$ Temp

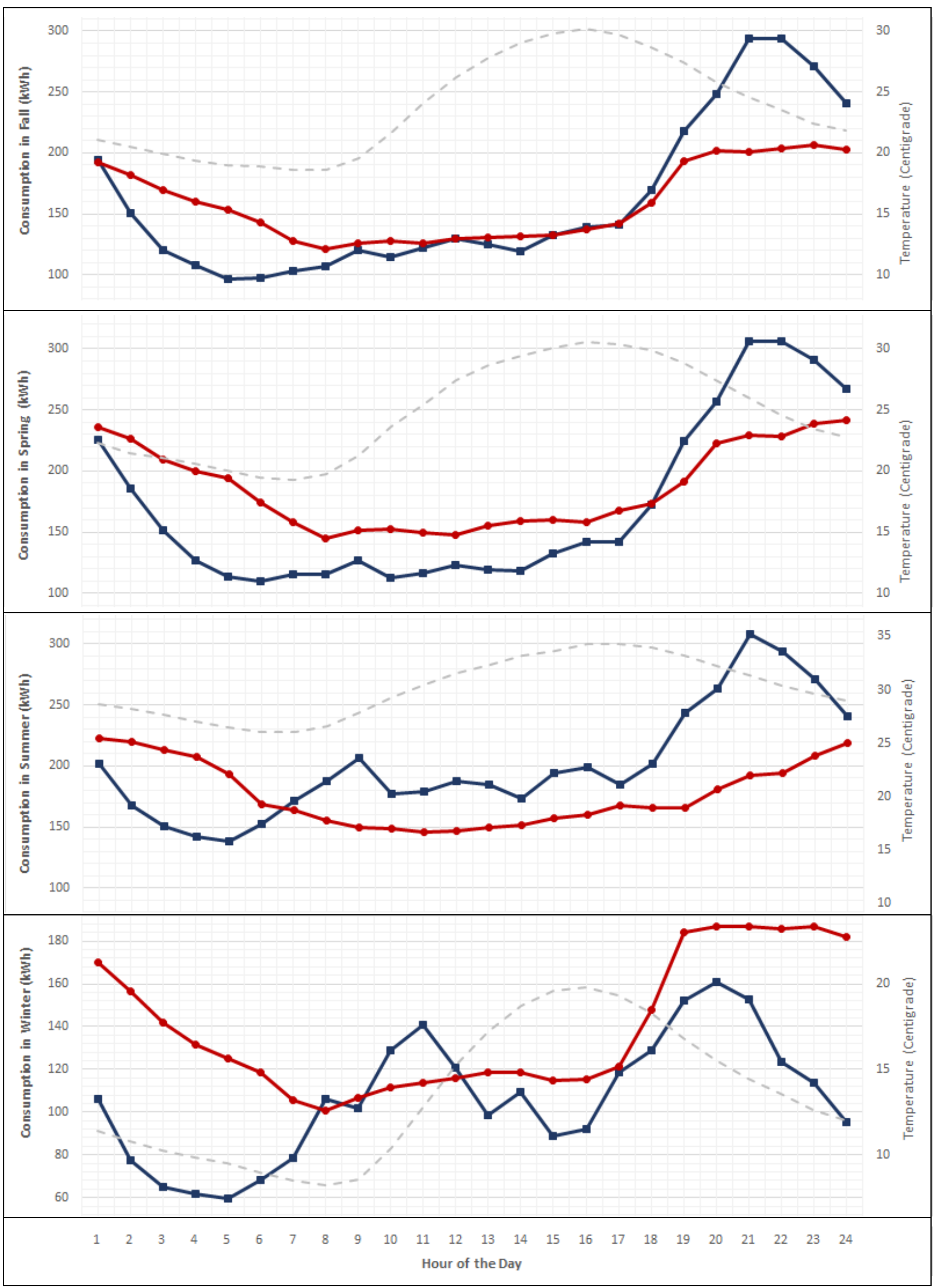

Figure 18: Season wise hourly consumption (a) Fall (b) Spring (c) Summer and (d) Winter. 


\subsection{Model Validation and Results}

It is quite difficult to perform the validation of Agent Based Models (ABM), because: (i) ABM are decentralized with no central control; (ii) ABM undergo bottom-up development of the individual entities; and (ii) The behavior in ABM are modeled in different hierarchical layers, e.g., in our case: Neighborhoods $\rightarrow$ Houses $\&$ Persons $\rightarrow$ Appliances. When the ABM is executed, a population of replicated agents is initialized and they interact with each other, where we can observe the emergent effect of all the interactions of different entities. Therefore, the aggregated results of the entities, rather than their individual results, are matched with the statistical effect of the whole system. The results of Mean Absolute Deviation (MAD), Root Mean Square Error (RMSE) Mean Absolute Percentage Error (MAPE\%) and Co-efficient of Variance of the Root Mean Square Error CV(RMSE) are calculated by using Equation 3 - 5. The results are showing that the mean percentage error of the four seasons lies in the range of $17 \%$ to $29 \%$, however, all the seasons are meeting the ASHRAE's tolerance of $30 \% \mathrm{CV}$ (RMSE) for hourly values.

$$
\boldsymbol{M A D}=\frac{\sum_{t=1}^{n}\left|A_{t}-F_{t}\right|}{n}
$$

Equation 3: Mean Absolute Deviation (MAD)

$$
\boldsymbol{R} \boldsymbol{M S} \boldsymbol{E}=\sqrt{\frac{\sum_{t=1}^{n}\left(A_{t}-F_{t}\right)^{2}}{n}}
$$

Equation 4: Root Mean Square Error (RMSE)

$$
\boldsymbol{M A P E}=\frac{\sum_{t=1}^{n}\left|\frac{A_{t}-F_{t}}{A_{t}}\right|}{n} \times 100
$$

Equation 5: Mean Absolute Percentage Error (MAPE)

$$
\boldsymbol{C V}(\boldsymbol{R} \boldsymbol{M S} \boldsymbol{E})=\frac{\sqrt{\frac{\sum_{t=1}^{n}\left(A_{t}-F_{t}\right)^{2}}{n-1}}}{\frac{\sum_{t=1}^{n} A_{t}}{n}} \times 100
$$

Equation 6: Co-efficient of Variance for Root Mean Square Error CV(RMSE)

Table 5: Validation Results

\begin{tabular}{|l|c|c|c|c|}
\hline Season & MAD & RMSE & MAPE \% & CV(RMSE) \% \\
\hline Fall & 30.11 & 37.26 & 18.26 & 22.55 \\
\hline Winter & 24.873 & 34.763 & 17.98 & 24.92 \\
\hline Spring & 39.61 & 50.09 & 20.56 & 25.76 \\
\hline Summer & 49.13 & 54.46 & 28.16 & 29.37 \\
\hline
\end{tabular}




\section{Analysis and Discussion}

The simulation results show that the model is correctly representing the actual site under observation (i.e., NUST Housing), as the deviation from the actual data in each season is within $30 \%$ and is acceptable as per the ASHRAE's criteria. The modelling exercise has provided us various insights which correlate with the real social behavior of the community under study. For instance, it can be observed from the simulations of all seasons that there is relatively less consumption during the morning period, and higher during evenings and nights. This reflects the behaviour commonly observed in the working-class community of Pakistan, since the model reflects a typical neighborhood in an urban area of the capital city of Pakistan. The proposed framework is fully capable to model communities where the consumption can be more during the day time, where there is always a parent / grandparent(s) at home or there is a joint family setup. Similarly, discrepancies in the simulation results are also explicable with the social behaviour. For example, we can observe from Figure 18 that, in the fall season, simulation has the least deviation. Whereas, simulation in the Summer season is showing highest deviation. This can be explained as follows: The simulated consumption is higher in summer because of the modeled behaviour, whereas the real data reflects less consumption because most of the families in the selected site travel during summer vacations. Moreover, the energy consumption is directly proportional to the increase in temperature, hence Spring and Summer seasons have higher energy consumption as compared to Winter and Fall, which is due to the tropical climate of Pakistan. That is why, we did not consider heating devices in colder seasons because the use of electricity for heating in winter is not common, and gas is mostly used instead.

In the light of the above discussion, and our experience in using our framework to simulate electricity consumption of an observed site (NUST housing), we assert that our approach is useful to synthesize household electricity demand profiles. The framework has a huge utility for the developing countries like Pakistan, because such countries lack the infrastructure of smart meters, especially in the residential sector. Therefore, the framework provides a low-cost solution to estimate power demand and provide decision support to the utility companies for effective energy planning. Furthermore, the researcher's community in the area of smart grids in Pakistan is in an immense need of such frameworks, due to the unavailability of the real data, to carry their research in developing control and management schemes for the future grids in Pakistan. For instance, to simulate the effectiveness of demand response in Pakistan, one needs multiple realizations of power demand, e.g., to completely formulate a power optimization problem. As there is an unavailability of actual consumption data, either physical models, as proposed in this paper, or data-based models constructed through such physical models can be used.

\section{Summary, Conclusion and Future Work}

In this paper, we proposed an agent-based modeling and simulation framework for the estimation of per minute electricity consumption of domestic consumers. We developed a hierarchical, multi-resolution, multiscale, dynamic, agent-based bottom-up model which calculates the per minute electricity consumption of a given number of households, by accumulating the consumption of major electrical appliances in the household. We currently implemented twelve (12) different types of appliances for four (4) different types of houses, with four (4) types of occupancy patterns. However, the framework is open-ended, and allows the users to: (i) add any number of appliances; (ii) modify types of houses with different configurations; and (iii) provides mechanism to add/modify occupancy patterns. We also incorporated different exogenous variables such as: temperature, seasonal variations and the type of the days i.e., weekdays and weekends. We presented a case study of 264 domestic consumers using the data of the housing area of the campus, to compare and evaluate the season-wise simulation behaviour of our proposed framework with the real-world data. The results exhibited a mean absolute percentage error of 17 to $29 \%$, and comply with the ASHRAE's tolerance of $30 \% \mathrm{CV}(\mathrm{RMSE})$. A correctly implemented and validated, agent-based simulation framework will support in: (i) Estimation of the future energy demands over a long-range future; (ii) Analysis of the underlying complex dynamic behaviour of the population in different conditions; (iii) Behavioral analysis to promote responsible use of energy by incorporating necessary policies; (iv) Effective planning of the production resources and taking effective decisions for mix strategy electricity generation; and (v) Devising effective demand response strategies to curtail peaking events and to minimize blackouts.

In future, we plan to perform a thorough validation of our simulator, at the appliance level using Pakistan Residential Electricity Consumption Dataset (PRECON) (65), to study the model stochastic and parameter 
sensitivity and to apply optimization techniques to achieve the minimum CV(RMSE) values. Our proposed framework is scale-able and can be configured for a sizeable number of houses to build larger neighborhoods, but requires high performance distributed simulation platforms, therefore we intend to deploy our simulator on a disturbed simulation platform for high performance and scalability. Furthermore, we also aim to extend our framework for other sectors including: commercial, industrial and agricultural, and integrate all these sectors to form a country-scale energy consumption model. Lastly, we plan to utilize the model for the implementation of the demand response strategies. The challenge includes the lack of communication and smart meter infrastructure on a scale that can actually contribute to nationwide problems, such as network overloading, blackouts etc. In this regard, the proposed modelling provides the best available insight on the consumption patterns, with an analysis on how and where flexibility can be exploited for demand side management.

Funding: This work is partially supported by the applied research grant project ARG-004, funded by the U.S.Pakistan Center for Advanced Studies in Energy, National University of Sciences and Technology, Pakistan

Imran Mahmood is currently working as Research Fellow at Brunel University, London. He has served as Assistant Professor at the Department of Computing, School of Electrical Engineering and Computer Science, National University of Sciences and Technology, (Pakistan). Imran earned his Masters and doctoral degrees in Computer Systems at the School of Information and Communication Technology (ICT), KTH-Royal Institute of Technology Sweden in 2007 and 2013 respectively. His current research interests are in applied modeling, simulation, analysis and visualization of complex systems. He was responsible for the Conceptualization, Data acquisition, Funding acquisition, Methodology, Project administration, Supervision, and Writing - original draft. He can be reached at imran.mahmood@ seecs.edu.pk

Quair-tul-ain is currently working as Deputy Director (MIS) in National Electric Power Regulatory Authority (NEPRA), Pakistan. She earned her Master's Degree in Computer Science from Islamia University, Pakistan and currently pursuing her degree of MS Computer Science from National University of Science and Technology (NUST). Her current research interests are modeling and simulation of complex energy systems, analysis and visualization of Big Data in Power Systems. She was responsible for the model development, simulation, validation, visualization and analysis of results. She can be reached at qain.pk@gmail.com.

Hasan Arshad Nasir is currently working as an Assistant Professor at the Department of Electrical Engineering in the School of Electrical Engineering and Computer Science, National University of Sciences and Technology, (Pakistan). Hasan earned his $\mathrm{PhD}$ from the Electrical Engineering department at the University of Melbourne in 2016. His current research interests are in the control and modelling of grid systems. He was responsible for the Formal analysis, Validation, Visualization, Writing review \& editing. He can be reached at hasan.nasir@seecs.edu.pk

Fahad Javed is Harvard South Asia Institute's Aman Fellow; Currently working as Assistant Professor at the Department of Computing, School of Electrical Engineering and Computer Science (SEECS), National University of Sciences and Technology, (Pakistan); Principal Investigator, "On building socio-economic what-if simulator for renewable energy technologies in Pakistan”, Pakistan Strategy Support Program by International Food Policy Research Institute; Earned Doctoral degree in Computer Systems at SBA School of Science and Engineering LUMS, Pakistan in 2014; Earned Master degree in Computer Systems at SBA School of Science and Engineering LUMS, Pakistan in 2010; Involved in research activities in demand side management and forecasting, modeling and simulation of household energy load for smart grids; He was responsible for the model validation and analysis of results. He can be reached at fahad.javed@seecs.edu.pk

José Antonio Aguado is Full Professor and Department Head at the Electrical Engineering Department, University of Málaga in Spain. He also serves regularly as consultant to Asian Development Bank and WorldBank on SmartGrids and Renewable Energy Projects. He has more than 40 publicly-funded research and consultancy projects in the area of operation and planning of smartgrids, renewable energies, energy efficiency, and wireless power transfer. He has authored 90+ papers in journals, book chapters, and conferences. He actively participates in scientific associations such as CIGRE and IEEE, where he was Vice-President of the IEEE Power and Energy Spanish Section. His expertise lies in: Operation and Planning of Electric Energy Systems Renewable Energy, Energy Storage and Electric Vehicles. He was responsible for the Conceptualization, Supervision and Writing - review \& editing. He can be reached at jaguado@uma.es

\section{References}

1. Kugelman M. Pakistan's Interminable Energy Crisis: Is There Any Way Out? Woodrow Wilson International Center for Scholars; 2015.

2. Ebinger CK. Energy and security in South Asia: cooperation or conflict? Brookings Institution Press; 2011.

3. Pruckner M, Eckhoff D, German R. Modeling Country-Scale Electricity Demand Profiles. In: Proceedings of the 2014 Winter Simulation Conference. IEEE Press; 2014. p. 1084-1095. (WSC '14).

4. Javed F, Arshad N, Wallin F, Vassileva I, Dahlquist E. Forecasting for demand response in smart grids: An 
analysis on use of anthropologic and structural data and short term multiple loads forecasting. Appl Energy. 2012;96:150-60.

5. Kim J-H, Shcherbakova A. Common failures of demand response. Energy. 2011;36(2):873-80.

6. Hayn M, Bertsch V, Fichtner W. Electricity load profiles in Europe: The importance of household segmentation. Energy Res Soc Sci. 2014;3:30-45.

7. Paatero J V, Lund PD. A model for generating household electricity load profiles. Int J energy Res. 2006;30(5):273-90.

8. NEPRA. State of Industry Report. Islamabad, Pakistan; 2016.

9. Boßmann T, Staffell I. The shape of future electricity demand: exploring load curves in 2050s Germany and Britain. Energy. 2015;90:1317-33.

10. Kremers EA. Modelling and simulation of electrical energy systems through a complex systems approach using agent-based models. KIT scientific publishing; 2013.

11. Zeyringer M, Andrews D, Schmid E, Schmidt J, Worrell E. Simulation of disaggregated load profiles and construction of a proxy-microgrid for modeling purposes. In: 2012 9th International Conference on the European Energy Market. 2012. p. 1-8.

12. Taylor S. Agent-based modeling and simulation. Springer; 2014.

13. Bonabeau E. Agent-based modeling: Methods and techniques for simulating human systems. Proc Natl Acad Sci. 2002;99(suppl 3):7280-7.

14. Alvi MSQ, Mahmood I, Javed F, Malik AW, Sarjoughian H. Dynamic behavioural modeling, simulation and analysis of household water consumption in an urban area: a hybrid approach. In: 2018 Winter Simulation Conference (WSC). 2018. p. 2411-22.

15. Ringler $\mathrm{P}$, Keles D, Fichtner W. Agent-based modelling and simulation of smart electricity grids and markets--a literature review. Renew Sustain Energy Rev. 2016;57:205-15.

16. Northeast TC. ASHRAE [Internet]. Atlanta, Georgia 30329 US. 2018. Available from: https://www.ashrae.org/technical-resources/standards-and-guidelines

17. Grandjean A, Adnot J, Binet G. A review and an analysis of the residential electric load curve models. Renew Sustain energy Rev. 2012;16(9):6539-65.

18. Richardson I, Thomson M, Infield D, Clifford C. Domestic electricity use: A high-resolution energy demand model. Energy Build. 2010;42(10):1878-87.

19. Heunis S, Dekenah M. A load profile prediction model for residential consumers in South Africa. In: Twenty-Second Domestic Use of Energy. 2014. p. 1-6.

20. Chuan L, Ukil A. Modeling and validation of electrical load profiling in residential buildings in Singapore. IEEE Trans Power Syst. 2014;30(5):2800-9.

21. Crawley DB, Lawrie LK, Winkelmann FC, Buhl WF, Huang YJ, Pedersen CO, et al. EnergyPlus: creating a new-generation building energy simulation program. Energy Build. 2001;33(4):319-31.

22. Wagner C, Waniek C, Häger U. Modeling of household electricity load profiles for distribution grid planning and operation. In: 2016 IEEE International Conference on Power System Technology (POWERCON). 2016. p. 1-6.

23. Bizzozero F, Gruosso G, Vezzini N. A time-of-use-based residential electricity demand model for smart grid applications. In: 2016 IEEE 16th International Conference on Environment and Electrical Engineering (EEEIC). 2016. p. 1-6.

24. Ali M, Iqbal MJ, Sharif M. Relationship between extreme temperature and electricity demand in Pakistan. 
Int J Energy Environ Eng. 2013;4(1):36.

25. Amara F, Agbossou K, Dubé Y, Kelouwani S, Cardenas A. Estimation of temperature correlation with household electricity demand for forecasting application. In: IECON 2016-42nd Annual Conference of the IEEE Industrial Electronics Society. 2016. p. 3960-5.

26. Latif S, Shabani A, Esser A, Martkovich A. Analytics of residential electrical energy profile. In: 2017 IEEE 30th Canadian Conference on Electrical and Computer Engineering (CCECE). 2017. p. 1-4.

27. Stankovic L, Stankovic V, Liao J, Wilson C. Measuring the energy intensity of domestic activities from smart meter data. Appl Energy. 2016;183:1565-80.

28. Ebrahim AF, Mohammed O. Household load forecasting based on a pre-processing non-intrusive load monitoring techniques. In: 2018 IEEE Green Technologies Conference (GreenTech). 2018. p. 107-14.

29. Coelho VN, Coelho IM, Coelho BN, Reis AJR, Enayatifar R, Souza MJF, et al. A self-adaptive evolutionary fuzzy model for load forecasting problems on smart grid environment. Appl Energy. 2016;169:567-84.

30. Ali D, Yohanna M, Puwu MI, Garkida BM. Long-term load forecast modelling using a fuzzy logic approach. Pacific Sci Rev A Nat Sci Eng. 2016;18(2):123-7.

31. Giasemidis G, Haben S, Lee T, Singleton C, Grindrod P. A genetic algorithm approach for modelling low voltage network demands. Appl Energy. 2017;203:463-73.

32. Raza MQ, Khosravi A. A review on artificial intelligence based load demand forecasting techniques for smart grid and buildings. Renew Sustain Energy Rev. 2015;50:1352-72.

33. Bustos-Turu G, van Dam KH, Acha S, Markides CN, Shah N. Simulating residential electricity and heat demand in urban areas using an agent-based modelling approach. In: 2016 IEEE International Energy Conference (ENERGYCON). 2016. p. 1-6.

34. Zheng M, Meinrenken CJ, Lackner KS. Agent-based model for electricity consumption and storage to evaluate economic viability of tariff arbitrage for residential sector demand response. Appl Energy. 2014;126:297-306.

35. Wang Y, Lin H, Liu Y, Sun Q, Wennersten R. Management of household electricity consumption under price-based demand response scheme. J Clean Prod. 2018;204:926-38.

36. Azar E, Nikolopoulou C, Papadopoulos S. Integrating and optimizing metrics of sustainable building performance using human-focused agent-based modeling. Appl Energy. 2016;183:926-37.

37. Zhang T, Siebers P-O, Aickelin U. Modelling electricity consumption in office buildings: An agent based approach. Energy Build. 2011;43(10):2882-92.

38. Lin H, Liu Y, Sun Q, Xiong R, Li H, Wennersten R. The impact of electric vehicle penetration and charging patterns on the management of energy hub--A multi-agent system simulation. Appl Energy. 2018;230:189_ 206.

39. Lin H, Wang Q, Wang Y, Wennerstern R, Sun Q. Agent-based modeling of electricity consumption in an office building under a tiered pricing mechanism. Energy Procedia. 2016;104:329-35.

40. Gaetani I, Hoes P-J, Hensen JLM. Occupant behavior in building energy simulation: Towards a fit-forpurpose modeling strategy. Energy Build. 2016;121:188-204.

41. Widén J, Wäckelgård E. A high-resolution stochastic model of domestic activity patterns and electricity demand. Appl Energy. 2010;87(6):1880-92.

42. Aerts D, Minnen J, Glorieux I, Wouters I, Descamps F. A method for the identification and modelling of realistic domestic occupancy sequences for building energy demand simulations and peer comparison. Build Environ [Internet]. 2014;75:67-78. Available from: 
http://www.sciencedirect.com/science/article/pii/S0360132314000304

43. McKenna E, Krawczynski M, Thomson M. Four-state domestic building occupancy model for energy demand simulations. Energy Build [Internet]. 2015;96:30-9. Available from: http://www.sciencedirect.com/science/article/pii/S0378778815002054

44. Flett G, Kelly N. A disaggregated, probabilistic, high resolution method for assessment of domestic occupancy and electrical demand. Energy Build [Internet]. 2017;140:171-87. Available from: http://www.sciencedirect.com/science/article/pii/S0378778817302591

45. Marshall E, Steinberger JK, Dupont V, Foxon TJ. Combining energy efficiency measure approaches and occupancy patterns in building modelling in the UK residential context. Energy Build [Internet]. 2016;111:98-108. Available from: http://www.sciencedirect.com/science/article/pii/S0378778815304060

46. Southampton U of. Occupancy Patterns Scoping Review Project [Internet]. 2016. Available from: https://www.google.com/url?sa=t\&rct=j\&q=\&esrc=s\&source=web\&cd=2\&ved=2ahUKEwjkupbkrZ_nAhV KilwKHb11C2IQFjABegQIAhAB\&url=https\%3A\%2F\%2Feprints.soton.ac.uk\%2F403390\%2F1\%2FFinal_R eport_-_Occupancy_Patterns_Scoping_Review_Project.pdf\&usg=AOvVaw1EiY081KDadRv231Q

47. Fischer D, Härtl A, Wille-Haussmann B. Model for electric load profiles with high time resolution for German households. Energy Build. 2015;92:170-9.

48. Shiraki H, Nakamura S, Ashina S, Honjo K. Estimating the hourly electricity profile of Japanese households - Coupling of engineering and statistical methods. Energy [Internet]. 2016;114:478-91. Available from: http://www.sciencedirect.com/science/article/pii/S0360544216311227

49. Hong T, Kim C-J, Jeong J, Kim J, Koo C, Jeong K, et al. Framework for Approaching the Minimum CV(RMSE) using Energy Simulation and Optimization Tool. Energy Procedia [Internet]. 2016;88:265-70. Available from: http://www.sciencedirect.com/science/article/pii/S1876610216302284

50. Glasgo B, Hendrickson C, Azevedo IL. Assessing the value of information in residential building simulation: Comparing simulated and actual building loads at the circuit level. Appl Energy [Internet]. 2017;203:348-63. Available from: http://www.sciencedirect.com/science/article/pii/S0306261917307237

51. Dehkordi NM, Baghaee HR, Sadati N, Guerrero JM. Distributed noise-resilient secondary voltage and frequency control for islanded microgrids. IEEE Trans Smart Grid. 2018;10(4):3780-90.

52. Afshari A, Karrari M, Baghaee HR, Gharehpetian GB, Karrari S. Cooperative Fault-Tolerant Control of Microgrids under Switching Communication Topology. IEEE Trans Smart Grid. 2019;

53. Baghaee HR, Mirsalim M, Gharehpetian GB. Power calculation using RBF neural networks to improve power sharing of hierarchical control scheme in multi-DER microgrids. IEEE J Emerg Sel Top Power Electron. 2016;4(4):1217-25.

54. Baghaee HR, Mirsalim M, Gharehpetian GB. Real-time verification of new controller to improve small/large-signal stability and fault ride-through capability of multi-DER microgrids. IET Gener Transm Distrib. 2016;10(12):3068-84.

55. Baghaee HR, Mirsalim M, Gharehpetian GB, Talebi HA. Eigenvalue, robustness and time delay analysis of hierarchical control scheme in multi-DER microgrid to enhance small/large-signal stability using complementary loop and fuzzy logic controller. J Circuits, Syst Comput. 2017;26(06):1750099.

56. Baghaee HR, Mirsalim M, Gharehpetian GB, Talebi HA. A new current limiting strategy and fault model to improve fault ride-through capability of inverter interfaced DERs in autonomous microgrids. Sustain Energy Technol Assessments. 2017;24:71-81.

57. Kumar P, Brar GS, Singh S, Nikolovski S, Baghaee HR, Balkić Z. Perspectives and Intensification of Energy 
Efficiency in Commercial and Residential Buildings Using Strategic Auditing and Demand-Side Management. Energies. 2019;12(23):4539.

58. Baghaee HR, Mirsalim M, Gharehpetan GB, Talebi HA. Nonlinear load sharing and voltage compensation of microgrids based on harmonic power-flow calculations using radial basis function neural networks. IEEE Syst J. 2017;12(3):2749-59.

59. Baghaee HR, Mirsalim M, Gharehpetian GB, Talebi HA. A Decentralized Robust Mixed $\$ H_{-}\{\{2\}\} /$ H_\{\{\{\infty \}\}\}\$ Voltage Control Scheme to Improve Small/Large-Signal Stability and FRT Capability of Islanded Multi-DER Microgrid Considering Load Disturbances. IEEE Syst J. 2018;12(3):2610-21.

60. Baghaee HR, Mirsalim M, Gharehpetian GB, Talebi HA. A decentralized power management and sliding mode control strategy for hybrid AC/DC microgrids including renewable energy resources. IEEE Trans Ind Informatics. 2017;

61. Baghaee HR, Mirsalim M, Gharehpetian GB, Talebi HA. Decentralized sliding mode control of WG/PV/FC microgrids under unbalanced and nonlinear load conditions for on-and off-grid modes. IEEE Syst J. 2017;12(4):3108-19.

62. McLoughlin F, Duffy A, Conlon M. Characterising domestic electricity consumption patterns by dwelling and occupant socio-economic variables: An Irish case study. Energy Build [Internet]. 2012;48:240-8. Available from: http://www.sciencedirect.com/science/article/pii/S0378778812000680

63. Yohanis YG, Mondol JD, Wright A, Norton B. Real-life energy use in the UK: How occupancy and dwelling characteristics affect domestic electricity use. Energy Build [Internet]. 2008;40(6):1053-9. Available from: http://www.sciencedirect.com/science/article/pii/S037877880700223X

64. Tsuji K, Sano F, Ueno T, Saeki O. Bottom-up simulation model for estimating end-use energy demand profiles in residential houses. In: Proc ACEEE summer study on energy efficiency in buildings. 2004.

65. Nadeem A, Arshad N. PRECON: Pakistan Residential Electricity Consumption Dataset. In: Proceedings of the Tenth ACM International Conference on Future Energy Systems [Internet]. New York, NY, USA: Association for Computing Machinery; 2019. p. 52-57. (e-Energy '19). Available from: https://doi.org/10.1145/3307772.3328317 